EMBRYARIDDLE
Aeronautical University

SCHOLARLY COMMONS

\section{International Journal of Aviation,} Aeronautics, and Aerospace

\title{
Experimental and Computational Evaluation of Five Hole Five Probe Flow Analyzer for Subsonic Wind Calibration
}

\author{
Akhila Rupesh Ms \\ Lovely Professional University, akhilarupesh56@gmail.com \\ J V Muruga Lal Jeyan Dr \\ Lovely Professional University, jvmllal@ymail.com
}

Follow this and additional works at: https://commons.erau.edu/ijaaa

Part of the Aerodynamics and Fluid Mechanics Commons

\section{Scholarly Commons Citation}

Rupesh, A., \& Jeyan, J. L. (2020). Experimental and Computational Evaluation of Five Hole Five Probe Flow Analyzer for Subsonic Wind Calibration. International Journal of Aviation, Aeronautics, and Aerospace, 7(4). https://doi.org/10.15394/ijaaa.2020.1523

This Concept Paper is brought to you for free and open access by the Journals at Scholarly Commons. It has been accepted for inclusion in International Journal of Aviation, Aeronautics, and Aerospace by an authorized administrator of Scholarly Commons. For more information, please contact commons@erau.edu. 


\section{Experimental and Computational Evaluation of Five Hole Five Probe Flow Analyzer for Subsonic Wind Calibration}

\section{Cover Page Footnote}

I would like to thank the Aerodynamics Laboratory, Department of Aerospace Engineering, Lovely Professional University, Punjab, India for providing the low speed subsonic wind tunnel facility for conducting the experiments. 
Wind tunnels are the ducts that allow the flow of air in axial direction over an object placed in the test section that has to be examined. Aerodynamic models are tested in the wind tunnel in order to know the flow patterns (Rupesh, Muruga lal Jeyan, Ram Mohan, et al., 2020). Wind tunnels are classified based on Mach Number in the test section and the shape of the tunnel. Based on variation in Mach Number, they are classified as subsonic $(\mathrm{M}<1)$, transonic $(\mathrm{M} \sim 1)$, supersonic $(2>\mathrm{M}<5)$, and hypersonic tunnels $(\mathrm{M}>5)$ (Rupesh \& MurugaLal Jeyan, 2020). Based on shape they are classified as open type, or close type wind tunnels. Generally, wind tunnel tests are done for two purposes - static stability and pressure test. Static stability test is carried out to find the forces acting on an object like side force, normal force, pitch, yaw, or roll moments (Pezzotti, 2011). Pressure test is carried out to analyse the external flow field by making some pressure tapings on the testing model. Before testing a model, calibration is must to ensure the accuracy of the desired output. Calibrating instruments are used to find the flow direction, rotation, and angularity (Gameiro Silva et al., 2003).

A pressure probe with multi probes is the measuring tool that has small holes in front of the instrument. It is used to measure the pressure of the fluid flow conditions in which the pressure is an essential factor for the operation of the system and calculates the static pressure, fluid pressure, and overall environmental pressure. When a body is involved in the flow of any fluid, the pressure distribution over its surface varies from a maximum at the stagnation point to some low values that are sometimes smaller than the static pressure far upstream and the pressure at different points on its surface is related to the direction and magnitude of the flow velocity (Paul et al., 2002). Most important is the effective and fast calibration of a multi-hole pressure probe. The multihole probes are easily inserted into any flowing fields without changing the direction of the flowing field, since the probes are small in size. A traditional probe contains a single cylindrical body at its tip with several holes (Lien \& Ahmed, 2011). The holes are internally attached to the device for pressure sensing. In order to determine the velocity magnitude and path of the probe, our program will interpret the pressure measured from each port when the probe is put in the flow.

Pressure holes with multiple holes has been deployed in many industrial and medical application considering its effectiveness and compactness (Yasar, \& Melda, 2011; Main, 2016). In the area of flow measurement, both in subsonic and supersonic areas pressure probes is having immense importance as far as research is considered. Hence more and more pressure measuring instruments are designed day by day for obtained more precise flow parameters (Rupesh et al., 2020).

If the instrument designed has even number of holes, it will be equally distributed on the circumference of the instrument. And if it holds an odd number of holes, one hole will be located at the central line to obtain stagnation values for the flow (Rupesh, MurugaLal Jeyan, \& Uthaman, 2020). 


\section{Methodology \\ Computational Method}

The five-hole five probe flow analyser has been designed in a way which allows it to be assembled and de assembled conveniently. The instrument is designed using CATIA so as to determine shock wave angle of specific category. The instrument has four major components namely Adapter, Wedge, Small Adapter, and Cone Probe. Figure 1 shows the top view, front view, and the isometric view of the assembled instrument. The five small probes are fitted on to the wedge with the small adaptor as a connection. The main adaptor is inserted from the back end which fits into the cavity of the edge. Apart from being convenient to assemble, this design facilitates ease in manufacturing each component.

\section{Figure 1}

Design of five-hole five probe flow analyser.
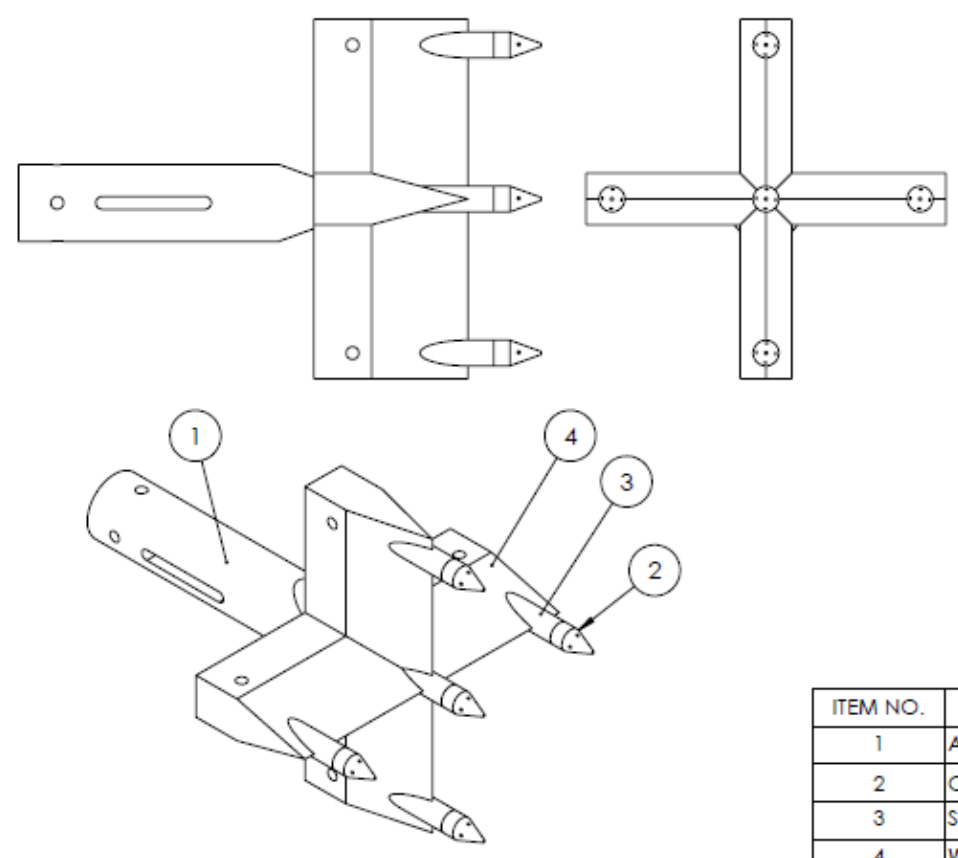

\begin{tabular}{|c|l|c|}
\hline ITEM NO. & \multicolumn{1}{|c|}{ PART NUMBER } & QTY. \\
\hline 1 & Adapter & 1 \\
\hline 2 & Cone Probe & 5 \\
\hline 3 & Small Adapter & 5 \\
\hline 4 & Wedge & 1 \\
\hline
\end{tabular}

As per the design consideration, the instrument has been manufactured using Aluminium Alloy 6061. The material selection has been done with comparing the material properties like strength to weight ratio and durability. Figure 2 shows the photographed view of the instrument parts manufactured as per the design and Figure 3 shows the final instrument ready for testing. 


\section{Figure 2}

Photographed view of five-hole five probe flow analyser before assembling.

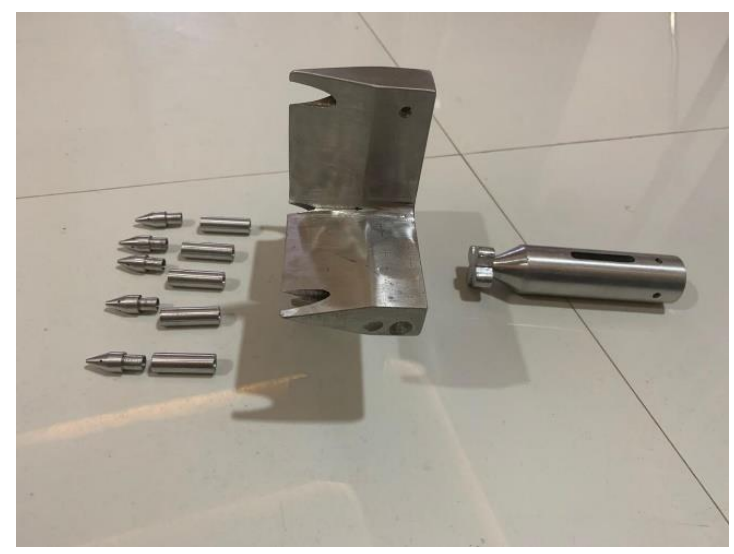

\section{Figure 3}

Photographed view of final five-hole five probe flow analyser.

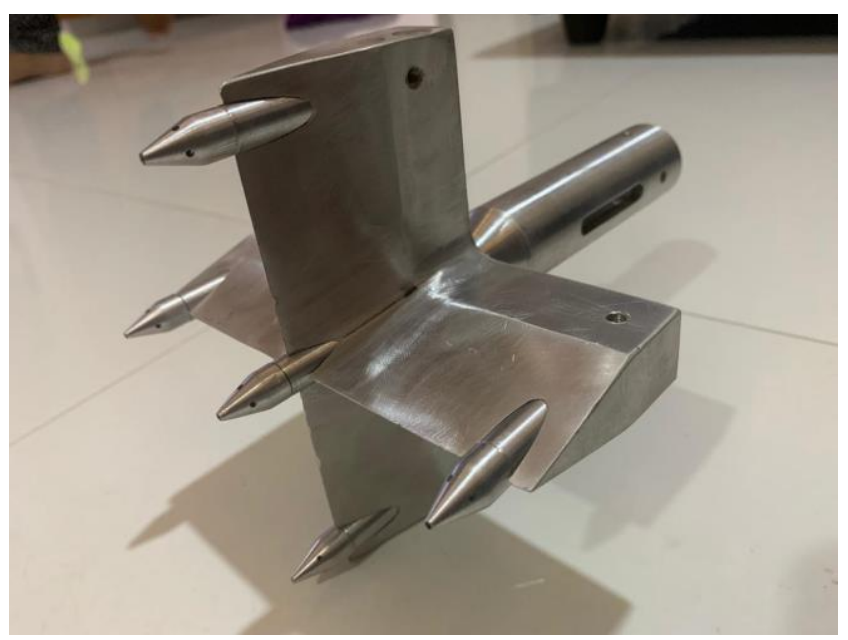

The designed instrument has been evaluated for its performance using CFD software (Ansys Fluent) in a two-dimensional method. The designed model is subjected to CFD analysis in order to obtain accurate results matching the practical conditions. At ambient pressure and temperature, the shock wave angle can be obtained for specific semi-cone angle and Mach Number using $\theta$ $\beta$-M relation.

$$
\tan \theta=2 \cot \beta\left[\frac{M_{1}^{2} \sin ^{2} \beta-1}{M_{1}^{2}(\gamma+\cos 2 \beta)+2}\right]
$$


For different values of semi-cone angle, shock wave angles have been determined using equation (1) corresponding to various Mach Numbers ranging from subsonic to supersonic regions. These values are incorporated with standard atmospheric conditions. The values so obtained has been formulated into a chart as shown in Figure 4.

\section{Figure 4}

Shock angles corresponding to various semi-cone angles at different Mach Numbers.

\begin{tabular}{|c|c|c|c|c|c|c|c|}
\hline \multirow[t]{2}{*}{ Mach no. } & \multicolumn{4}{|c|}{ Semi cone angle } & \multicolumn{3}{|c|}{$=40^{\circ}$ cone angle } \\
\hline & $\theta=5$ & 10 & 15 & $20 \mathrm{~b}$ & 25 & 30 & \\
\hline 1.05 & 72.4 & - & - & -- & - & - & \\
\hline 1.1 & 65.6 & 67.0 & - & - & - & - & \\
\hline 1.2 & 56.4 & 57.5 & 60.6 & 72.5 & $=$ & - & \\
\hline 1.3 & 50.5 & 51.4 & 53.4 & 58.0 & - & - & \\
\hline 1.4 & 45.5 & 46.3 & 48.3 & 52.8 & 59.3 & - & \\
\hline 1.6 & 39.0 & 39.4 & 41.6 & 46.2 & 52.2 & 59.1 & 8 \\
\hline 1.8 & 34.0 & 34.6 & 37.1 & 41.6 & 46.7 & 52.6 & \\
\hline 2 & 30.1 & 31.3 & 33.7 & 38.0 & 43.0 & 48.3 & 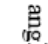 \\
\hline 2.5 & 23.8 & 24.8 & 27.8 & 32.2 & 37.1 & 42.6 & \\
\hline 3.0 & 20.0 & 21.3 & 24.7 & 29.3 & 34.2 & 39.5 & \\
\hline 3.5 & 16.9 & 19.4 & 23.4 & 27.7 & 32.7 & 38.3 & \\
\hline
\end{tabular}

From the chart obtained with $\theta-\beta-\mathrm{M}$ relation, it has been noted that for semi-cone angle of $20^{\circ}$, the shock wave angle approached $29.3^{\circ}$ at the Mach Number range of 3 . Hence it has to be concluded that the instrument designed will be suitable to operate in a region of subsonic Mach Number starting from 1.1 to supersonic Mach of maximum 3, beyond which the shock waves generated may hit the probe and results cannot be generated. Furthermore, the semi- cone angle cannot be increased since it will have a very strong negative impact on tunnel blockage factor and sensitivity of the instrument.

\section{Experimental Method}

The instrument has been mounted in the wind tunnel using nulling method. The procedure of mounting the instrument involves placing the instrument in the tunnel test section parallel to the tunnel axis. The pressure probes are connected with the multi tube manometer. At various velocities the instrument has to be tested and evaluated. Initially it is to be made sure that the instrument is exactly kept parallel to the tunnel axis. The instrument is fixed at various location on the wind tunnel and the values so obtained are recorded. Parallel to the instrument mounted, a Pitot static tube is also connected to a sensor in order to find the dynamic pressure of the flow. The specification of the tunnel used for testing the instrument is given in Table 1. 
Table 1

Wind tunnel specification

\begin{tabular}{ll}
\hline Item & Specification \\
\hline Organisation & Lovely Professional University \\
Test Section Size & $600 \mathrm{~mm} \times 600 \mathrm{~mm}$ \\
Maximum Velocity & $75 \mathrm{~m} / \mathrm{s}$ \\
Contraction Ratio & $9: 1$ \\
Power Supply & 3 Phase AC - 400 W-64 Amps
\end{tabular}

The pressure tapings have been numbered $1,2,3 \ldots \ldots \ldots \ldots . .25$ and the probes are named as Probe A, Probe B, Probe C, Probe D, and Probe E. The instrument is mounted in the identified wind tunnel and the obtained values of pressure are charted out and the coefficient of pressures are calculated. The numbering and naming of the pressure probes are as shown in Figure 5. 
Figure 5

Naming and numbering of pressure probes.

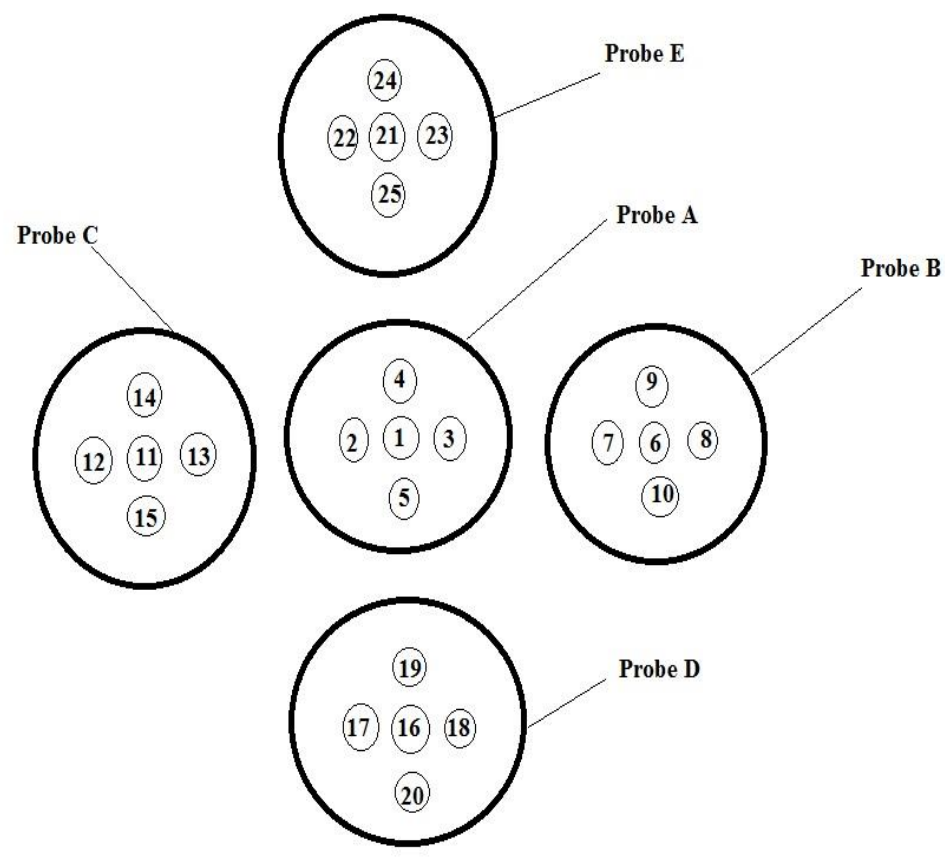

The formulae listed below are used to determine pressure coefficients for Probes A, B, C, D and E. For Probe A, for determining pressure coefficients, the following formulae are used.

$$
\begin{gathered}
C_{P_{\text {yaw }}=\frac{P_{2}-P_{3}}{P_{1}-\bar{P}}} \\
C_{P_{\text {pitch }}=\frac{P_{5}-P_{4}}{P_{1}-\bar{P}}} \\
C_{P_{\text {total }}=\frac{P_{1}-P_{\text {total }}}{P_{1}-\bar{P}}} \\
C_{P_{\text {static }}=\frac{\bar{P}-P_{\text {static }}}{P_{1}-\bar{P}}} \\
\bar{P}=\frac{P_{2}+P_{3}+P_{4}+P_{5}}{4}
\end{gathered}
$$

For Probe B, for determining pressure coefficients, the following formulae are used.

$$
\begin{gathered}
C_{P_{y a w}}=\frac{P_{7}-P_{8}}{P_{6}-\bar{P}} \\
C_{P_{\text {pitch }}}=\frac{P_{10}-P_{9}}{P_{6}-\bar{P}}
\end{gathered}
$$




$$
\begin{gathered}
C_{P_{\text {total }}=\frac{P_{6}-P_{\text {total }}}{P_{6}-\bar{P}}} \\
C_{P_{\text {static }}=\frac{\bar{P}-P_{\text {static }}}{P_{6}-\bar{P}}} \\
\bar{P}=\frac{P_{7}+P_{8}+P_{9}+P_{10}}{4}
\end{gathered}
$$

For Probe $\mathrm{C}$, for determining pressure coefficients, the following formulae are used.

$$
\begin{gathered}
C_{P_{\text {yaw }}=\frac{P_{12}-P_{13}}{P_{11} \overline{\bar{P}}}} \\
C_{P_{\text {pitch }}=\frac{P_{15}-P_{14}}{P_{11}-\bar{P}}} \\
C_{P_{\text {total }}=\frac{P_{11}-P_{\text {total }}}{P_{11}-\bar{P}}} \\
C_{P_{\text {static }}=\frac{\bar{P}-P_{\text {static }}}{P_{11}-\bar{P}}} \\
\bar{P}=\frac{P_{12}+P_{13}+P_{14}+P_{15}}{4}
\end{gathered}
$$

For Probe D, for determining pressure coefficients, the following formulae are used.

$$
\begin{gathered}
C_{P_{\text {yaw }}=\frac{P_{17}-P_{18}}{P_{16}-\bar{P}}} \\
C_{P_{\text {pitch }}=\frac{P_{20}-P_{19}}{P_{16}-\bar{P}}} \\
C_{P_{\text {total }}=\frac{P_{16}-P_{\text {total }}}{P_{16}-\bar{P}}} \\
C_{P_{\text {static }}=\frac{\bar{P}-P_{\text {static }}}{P_{16}-\bar{P}}} \\
\bar{P}=\frac{P_{17}+P_{18}+P_{19}+P_{20}}{4}
\end{gathered}
$$

For Probe E, for determining pressure coefficients, the following formulae are used.

$$
\begin{gathered}
C_{P_{\text {yaw }}=\frac{P_{22}-P_{23}}{P_{21} \overline{\bar{P}}}} \\
C_{P_{\text {pitch }}=\frac{P_{25}-P_{24}}{P_{21}-\bar{P}}} \\
C_{P_{\text {total }}=\frac{P_{21}-P_{\text {total }}}{P_{21}-\bar{P}}} \\
C_{P_{\text {static }}=\frac{\bar{P}-P_{\text {static }}}{P_{21}-\bar{P}}} \\
\bar{P}=\frac{P_{22}+P_{23}+P_{24}+P_{25}}{4}
\end{gathered}
$$




\section{D Analysis}

\section{Results and Discussion}

For $2 \mathrm{D}$ analysis, a single probe is taken so as to analyse the results obtained with the real time values obtained using $\theta-\beta-\mathrm{M}$ relation. The $2 \mathrm{D}$ analysis is done with varying the Mach Number at 1.01325 bar (ambient pressure) and $288 \mathrm{~K}$ (ambient temperature). The Mach Number provided for the analysis varies from subsonic to supersonic range. Mach Number starting from 0.5 has been given with an interval of 0.3 till supersonic Mach of 2.6 to validate the results obtained by $\theta-\beta-\mathrm{M}$ relation.

Figure 6 describes the static pressure change at subsonic speeds where Mach Number is 0.5. Here the air flow gets detached from surface profile of probe making a large Mach angle, due to which all pressure gets piled up at the front side of the probe, which is shown with the colour yellow. Figure 7 describes the temperature profile at Mach Number 0.5. As the velocity gets increased, there would be slit change in temperature. It is shown in colour green.

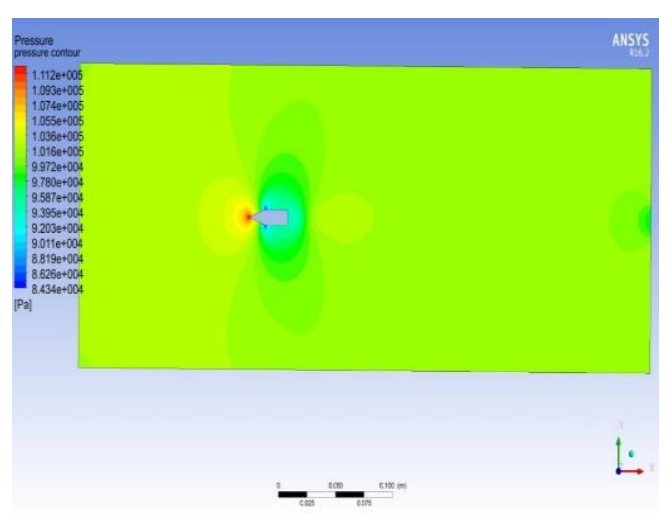

Figure 6. Static Pressure at Mach 0.5

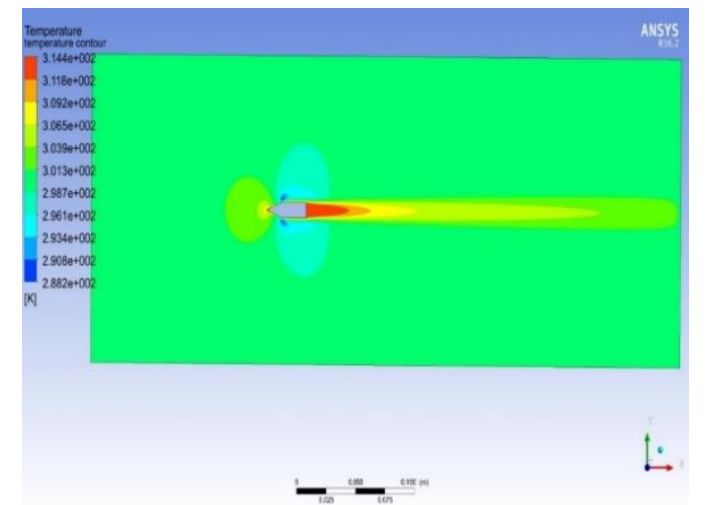

Figure 7. Static Temperature at Mach 0.5

Figure 8 describes the static pressure variation at Mach Number 0.8. Here the flow is not aligned or fitted with the probe surface which is same as Figure 6. In Figure 9, the green colour indicates the static temperature change at Mach Number 0.8. 


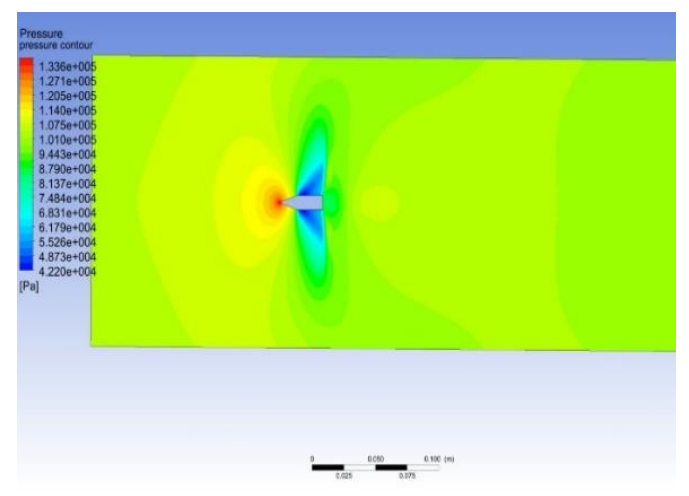

Figure 8. Static Pressure at Mach 0.8

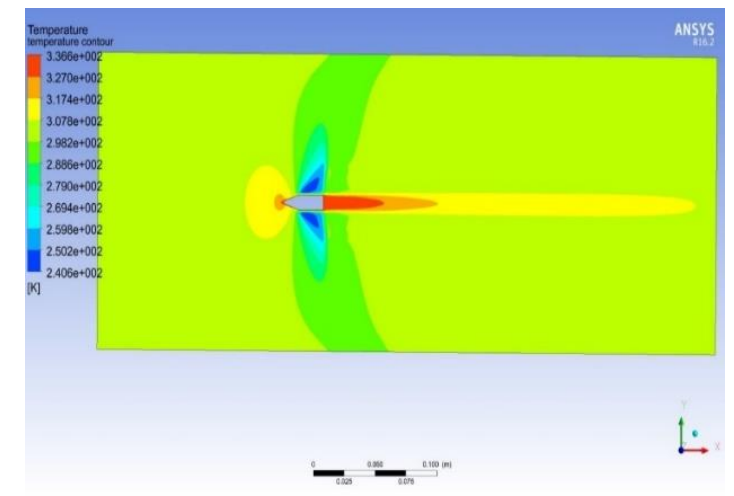

Figure 9. Static Temperature at Mach 0.8

Figure 10 indicates the static pressure variation at Mach Number 1.1. Here even the pressure gets accumulated at the front part of the probe which is shown in yellow colour by the shock wave formation. The profile of Figure 11 remains same as the Figure 10 with the slight variation in temperature by the increasing in Mach number.

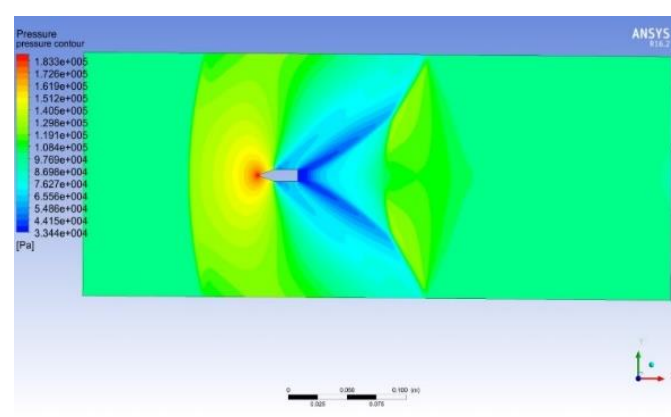

Figure 10. Static Pressure at Mach 1.1

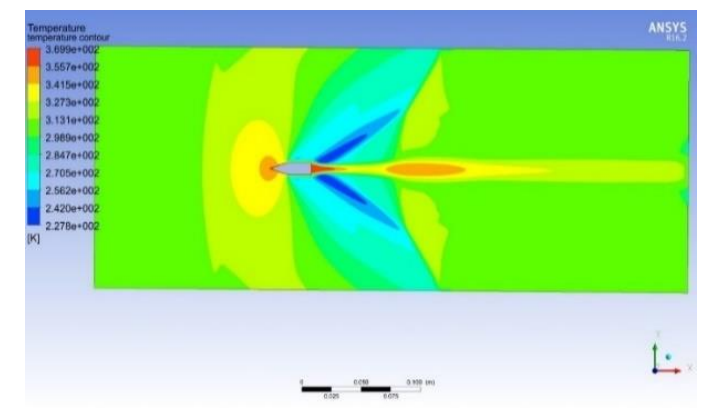

Figure 11. Static Temperature at Mach 1.1

In Figure 12, with the increase in Mach Number, there would be a formation of shock angle with the probe. The geometry of this wave can be differentiated with that of Figure 10. Figure 13 shows the change in temperature with minimal change in velocity. 


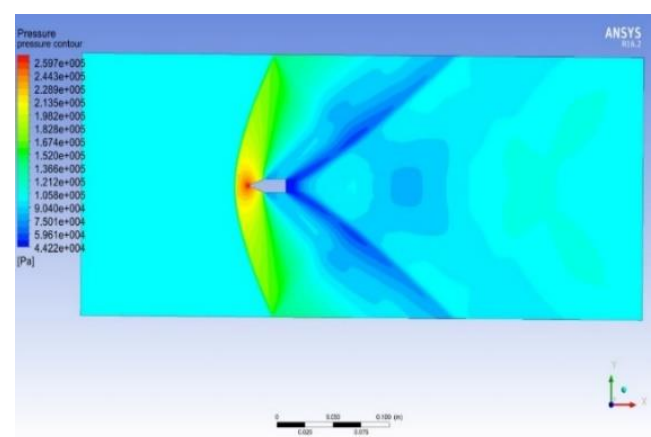

Figure 12. Static Pressure at Mach 1.4

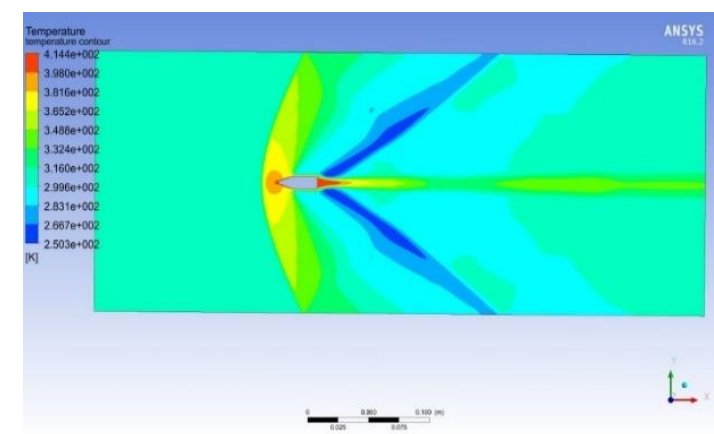

Figure 13. Static Temperature at Mach 1.4

Figure 14 shows the static pressure variation at Mach Number 1.7. Here, the Mach angle is forms with the probe where the pressure is perfectly fitted with the front tip of the probe. Figure 15 describes the temperature change at Mach 1.7 there would be increasing temperature with increasing velocity.

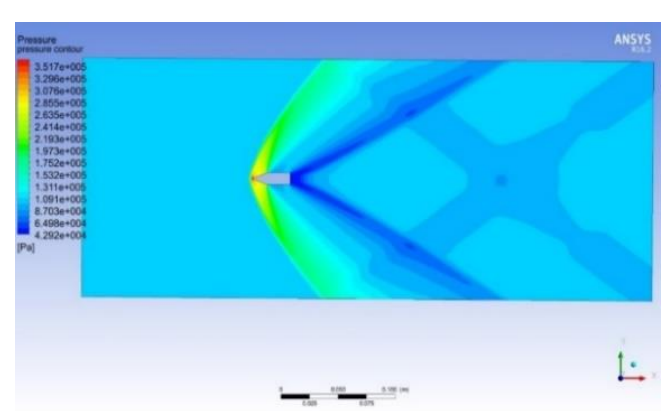

Figure 14. Static Pressure at Mach 1.7

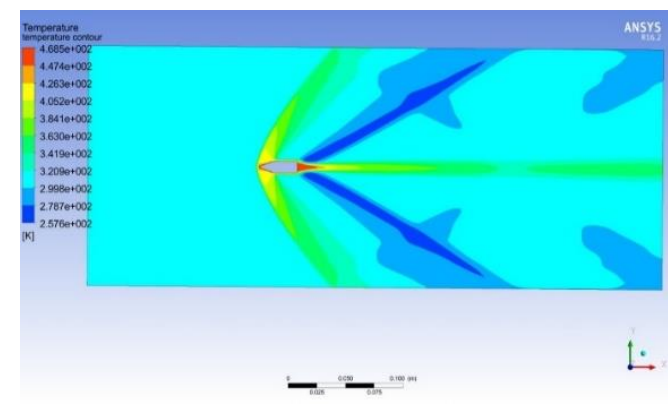

Figure 15. Static Temperature at Mach 1.7

Figure 16 describes the static pressure variation at Mach Number 2. With the increase in Mach Number, Mach angle get decreased. It is shown with the colour blue. Figure 17 describes the static temperature variation at Mach Number 2. This variation shown with the colour blue.

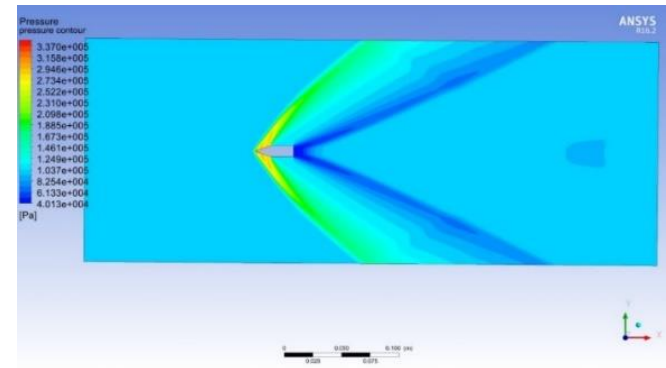

Figure 16. Static Pressure at Mach 2

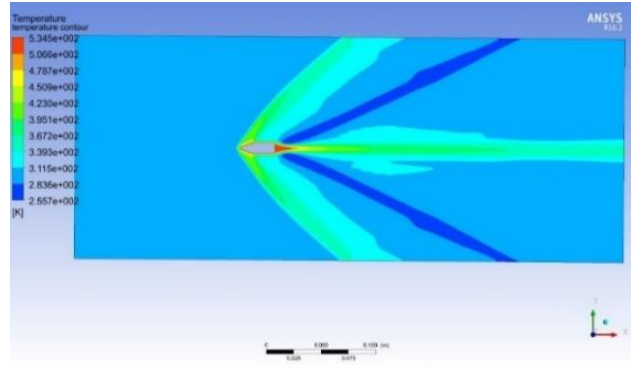

Figure 17. Static Temperature at Mach 2 
Figure 18 describes the static pressure variation at Mach Number 2.3. The Mach angle is further decreased and converges to the cone of the probe. Figure 19 describes the static temperature variation at Mach Number 2.3. The thick blue colour shows the change in temperature with increasing in Mach Number.

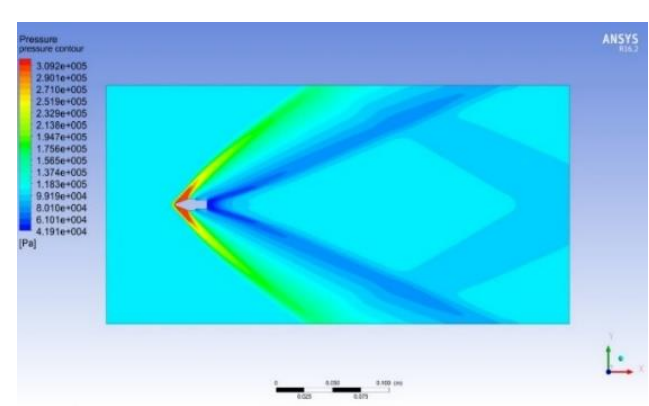

Figure 18. Static Pressure at Mach 2.3

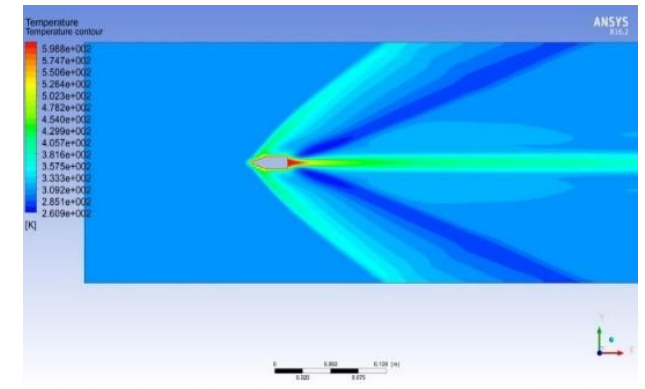

Figure 19. Static Temperature at Mach 2.3

Figure 20 describes the static pressure variation at Mach Number 2.6. The Mach angle decreases and converges more to the cone compared with Figure 18. The lower the shock angle, the more effectively the probe works. In Figure 21, the static temperature variation at Mach Number 2.6 is shown with the colour blue.

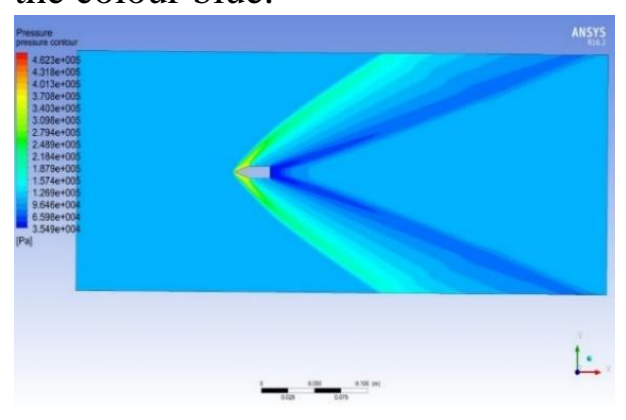

Figure 20. Static Pressure at Mach 2.6 Temperature at Mach 2

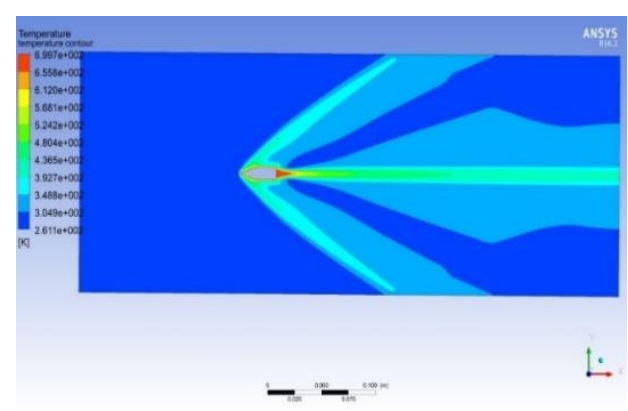

Figure 21. Static 


\section{Experimental Method}

The instrument has been mounted in the subsonic wind tunnel and tested for various subsonic velocities and recorded. The pressure and pressure coefficients values obtained at $34.4 \mathrm{~m} / \mathrm{s}$ for Probe A, B, C, D, and E are charted in Table 2 to Table 11 respectively refer to Appendix 1).

The results obtained by testing the five-hole five probe flow analyser at $34.4 \mathrm{~m} / \mathrm{s}$ is compared with the theoretical values of flow parameters. The theoretical values can be obtained by the formula of Mach Number, Dynamic Pressure, Stagnation temperature and Stagnation Pressure as given by Equation (2), (3), (4) and (5) respectively at $\gamma=1.4$. Later on, obtaining theoretical values of stagnation pressure and dynamic pressure, static pressure can be calculated by taking their difference. The results so obtained is charted in Table 12 .

$$
\begin{aligned}
& \text { Mach Number }=\frac{\text { Velocity of the fluid }(\mathrm{m} / \mathrm{s})}{\text { Velocity of Sound }(\mathrm{m} / \mathrm{s})} \\
& \text { Dynamic Velocity }=\frac{1}{2} \rho_{\infty} V_{\infty}^{2} \ldots \ldots \ldots \ldots \ldots \ldots
\end{aligned}
$$

Stagnation temperature, $\mathrm{T}_{0}=\mathrm{T}\left(1+\frac{\gamma+1}{2} M^{2}\right)$

Stagnation pressure, $\mathrm{P}_{0}=\rho \mathrm{RT}_{0}$

Table 12

\begin{tabular}{|c|c|c|c|c|c|}
\hline $\begin{array}{c}\text { Mach } \\
\text { no }\end{array}$ & $\begin{array}{c}\text { Static } \\
\text { Temperature } \\
(K)\end{array}$ & $\begin{array}{c}\text { Dynamic } \\
\text { pressure } \\
\left(\mathrm{N} / \mathrm{m}^{2}\right)\end{array}$ & $\begin{array}{c}\text { Stagnation } \\
\text { temperature } \\
(K)\end{array}$ & $\begin{array}{l}\text { Stagnation } \\
\text { pressure } \\
\left(\mathrm{N} / \mathrm{m}^{2}\right)\end{array}$ & $\begin{array}{c}\text { Static } \\
\text { pressure } \\
\left(\mathrm{N} / \mathrm{m}^{2}\right)\end{array}$ \\
\hline 0.1 & 294 & 724.808 & 294.5881482 & 103569.8282 & 102845.0202 \\
\hline
\end{tabular}

Theoretical values obtained for various flow parameters at $34.4 \mathrm{~m} / \mathrm{s}$

It is clearly evident that the results obtained in Tables 2 to 11 are matching with the theoretical results obtained in Table 12. Hence it is proved that the designed five-hole five probe flow analyser can be used as a successful instrument for subsonic wind tunnel calibration.

\section{Conclusion}

The manufactured five-hole five probe flow analyser as per the specified dimensions modelled in CATIA is tested and analysed for its performance and effectiveness using two-dimensional Computational Fluid Dynamics software. As per the performance evaluation, it is noted that the shock wave angle is getting decreased as the Mach Number increases from subsonic to supersonic level. In the analysis it is very clear that the instrument is suitable to be used till 
the Mach Number range of 3. Beyond this Mach Number, the proposed instrument may not be suitable since the shock wave angle and semi cone angles almost reaches the same range. Hence further increasing the Mach Number will not give suitable results for determining the flow parameters. Even the theoretical results obtained by $\theta-\beta-\mathrm{M}$ relation and the computational results obtained by CFD software analysis are also matching which proves the proposed instrument can be used for further experiments the field of fluid dynamics. A methodological evaluation for performance of the five-hole five probe flow analyser has been conducted at various Mach Number in subsonic wind tunnel. From the results charted during experimentation, it is crystal clear that the proposed instrument is very effective in determining the flow angularities inside the test section of any wind tunnel. The validation of the innovative flow analyser has thus been done effectively. It is concluded from the results obtained that the level of turbulence in the test section of the tunnel is almost normal everywhere apart from slight variations and fluctuations at some points in the tunnel. This data charted for various tunnel in this paper can be used for assisting experimentations done during practical real time applications. A perfect idea about the truth flow and performance of tunnel stated in the paper and detailed understanding about the flow parameters throughout the test section has been obtained. 


\section{References}

Gameiro Silva, M. C., Pereira, C. A. C., \& Cruz, J. M. S. (2003). On the use of a linear interpolation method in the measurement procedure of a sevenhole pressure probe. Journal of Thermal and Fluid Science, 28. Retrieved from https://doi.org/10.1016/S0894-1777(03)00074-8

Lien, S. J., \& Ahmed, N. A. (2011). An examination of suitability of multi-hole pressure probe technique for skin friction measurement in turbulent flow, Journal of Flow Measurement and Instrumentation, 22. Retrieved from 10.1016/j.flowmeasinst.2011.01.004

Main, J., Day, C. R. B., G., Lock, G. D., \& Oldfield, M. L. G. (2016). Calibration of a four-hole pyramid probe and area traverse measurements in a short-duration transonic turbine cascade tunnel. International Journal of Innovative Research in Science, Engineering and Technology, 1. Retrieved from 10.1007/BF00190681

Paul, A. R., Upadhyay, R. R., \& Jain, A. (2002). A novel calibration algorithm for five-hole pressure probe. Journal of Flow Measurement and Instrumentation, 3(2). Retrieved from 10.4314/ijest.v3i2.68136

Pezzotti, S., Juan, I. D., Nadal-Mora, V., \& Pesarini, A. (2011). A wind tunnel for anemometer calibration in the range of $0.2-1.25 \mathrm{~m} / \mathrm{s}$. Journal of Flow Measurement and Instrumentation, 22. Retrieved from https://doi.org/10.1016/j.flowmeasinst.2011.04.006

Rupesh, A., \& MurugaLal Jeyan, J. V. (2020). Performance evaluation of a two hole and five hole Flow analyzer for subsonic flow. International Journal of Advanced Science and Technology, 29(5), Retrieved from http://sersc.org/journals/index.php/IJAST/article/view/18243/9272

Rupesh, A., Muruga lal Jeyan, J. V., Ram Mohan, V. M., Kumar, P., Abhishek, T. Ashish, T., Reddy, K. V. V. M., \& Maddireddy, G. (2020) .Comparative study on wind tunnel calibrating instruments. Advances in Metrology and Measurement of Engineering Surfaces: Lecture Notes in Mechanical Engineering book series (LNME) - Springer, Singapore, 2. Retrieved from https://doi.org/10.1007/978-981-15-5151-2_14

Rupesh, A., Muruga lal Jeyan, J., \& Uthaman, S. (2020). Design and analysis of five probe flow analyser for subsonic and supersonic wind tunnel calibration. IOP Conference Series: Materials Science and Engineering, 1(715), Retrieved from 10.1088/1757-899X/715/1/012083

Yasar, M., \& Melda, O. C. (2011). A multi-tube pressure probe calibration method for measurements of mean flow parameters in swirling flows. Journal of Flow Measurement and Instrumentation, 9. Retrieved from 10.15680/IJIRSET.2016.0504040 


\section{Appendix}

Table 2

Pressure values for probe A at $34.4 \mathrm{~m} / \mathrm{s}$

\begin{tabular}{|c|c|c|c|c|c|c|}
\hline Coordinate & $P_{1}$ & $P_{2}$ & $P_{3}$ & $P_{4}$ & $P_{5}$ & $\bar{P}$ \\
\hline$(0,0,0)$ & 99981.6 & 99648.2 & 99596.3 & 99787.6 & 99789.7 & 99705.45 \\
\hline$(50,0,0)$ & 99979.4 & 99646.3 & 99592.4 & 99785.3 & 99787.4 & 99702.85 \\
\hline$(50,50,0)$ & 99980.4 & 99648.5 & 99595.5 & 99784.5 & 99786.3 & 99703.7 \\
\hline$(50,100,0)$ & 99984.7 & 99644.2 & 99597.1 & 99786.6 & 99785.1 & 99703.25 \\
\hline$(50,150,0)$ & 99982 & 99648.2 & 99596.6 & 99782.7 & 99789.2 & 99704.18 \\
\hline$(100,0,0)$ & 99978.6 & 99649.8 & 99593 & 99783.9 & 99784.6 & 99702.93 \\
\hline$(100,50,0)$ & 99985.2 & 99647.1 & 99593.7 & 99781.8 & 99782.7 & 99701.33 \\
\hline$(100,100,0)$ & 99982.6 & 99647 & 99597.6 & 99784 & 99788.8 & 99704.18 \\
\hline$(100,150,0)$ & 99978.1 & 99644.7 & 99594.1 & 99788.7 & 99782.7 & 99702.55 \\
\hline$(150,0,0)$ & 99981.5 & 99647.6 & 99599.4 & 99781.9 & 99781.5 & 99702.6 \\
\hline$(150,50,0)$ & 99978 & 99642.3 & 99597.3 & 99782.3 & 99785.3 & 99701.8 \\
\hline$(150,100,0)$ & 99983.6 & 99646.1 & 99596.7 & 99789.1 & 99785.7 & 99704.4 \\
\hline$(150,150,0)$ & 99981.7 & 99647.3 & 99594.3 & 99788.2 & 99787.2 & 99704.25 \\
\hline$(-50,0,0)$ & 99980.9 & 99644.4 & 99597.6 & 99789 & 99785.7 & 99704.08 \\
\hline$(-50,-50,0)$ & 99978.4 & 99646.6 & 99593.6 & 99786.4 & 99782.8 & 99702.35 \\
\hline$(-50,-100,0)$ & 99981.3 & 99647.3 & 99595.2 & 99783.4 & 99786.5 & 99703.1 \\
\hline$(-50,-150,0)$ & 99980.7 & 99646 & 99593.7 & 99786.1 & 99783.7 & 99702.4 \\
\hline$(-100,0,0)$ & 99982.7 & 99647.7 & 99599.1 & 99785.2 & 99789.2 & 99705.3 \\
\hline$(-100,-50,0)$ & 99981.1 & 99643.6 & 99593.4 & 99785.4 & 99787.6 & 99702.5 \\
\hline$(-100,-100,0)$ & 99983.7 & 99646.3 & 99594.6 & 99786 & 99781.9 & 99702.28 \\
\hline$(-100,-150,0)$ & 99980 & 99645.4 & 99591 & 99782.9 & 99784.5 & 99701.05 \\
\hline$(-150,0,0)$ & 99984.8 & 99643 & 99597.2 & 99783.5 & 99786.3 & 99702.6 \\
\hline$(-150,-50,0)$ & 99981.5 & 99647.6 & 99593.5 & 99786.2 & 99789.8 & 99704.28 \\
\hline$(-150,-100,0)$ & 99983.4 & 99647.8 & 99596.3 & 99787.4 & 99787.75 & 99704.81 \\
\hline$(-150,-150,0)$ & 99982 & 99646.3 & 99595 & 99788.9 & 99782.9 & 99703.33 \\
\hline$(50,0,50)$ & 99983.7 & 99647.1 & 99594.1 & 99783.9 & 99784.6 & 99702.43 \\
\hline$(50,50,50)$ & 99981.4 & 99646.6 & 99599.4 & 99781.8 & 99782.7 & 99702.63 \\
\hline$(50,100,50)$ & 99983.1 & 99644.7 & 99597.3 & 99783.7 & 99785.1 & 99702.7 \\
\hline$(50,150,50)$ & 99978.7 & 99647.6 & 99596.7 & 99788.7 & 99789.2 & 99705.55 \\
\hline$(100,0,50)$ & 99982.9 & 99642.3 & 99594.3 & 99785.3 & 99784.6 & 99701.63 \\
\hline$(100,50,50)$ & 99978.2 & 99646.1 & 99597.6 & 99789.1 & 99782.7 & 99703.88 \\
\hline$(100,100,50)$ & 99983.4 & 99647.3 & 99593.6 & 99783.9 & 99788.8 & 99703.4 \\
\hline$(100,150,50)$ & 99981.6 & 99644.4 & 99595.2 & 99781.8 & 99782.7 & 99701.03 \\
\hline$(150,0,50)$ & 99983.2 & 99646.6 & 99593.7 & 99784 & 99781.5 & 99701.38 \\
\hline$(150,50,50)$ & 99981 & 99647.3 & 99599.1 & 99788.7 & 99785.3 & 99705.1 \\
\hline
\end{tabular}




\begin{tabular}{|c|c|c|c|c|c|c|}
\hline$(150,100,50)$ & 99983.7 & 99648.2 & 99593.4 & 99785.3 & 99787.4 & 99703.58 \\
\hline$(150,150,50)$ & 99979.6 & 99646.3 & 99594.6 & 99789.1 & 99786.3 & 99704.08 \\
\hline$(-50,0,-50)$ & 99981.1 & 99648.5 & 99591.4 & 99788.2 & 99785.1 & 99703.3 \\
\hline$(-50,-50,-50)$ & 99980.6 & 99644.2 & 99597 & 99788.6 & 99789.2 & 99704.8 \\
\hline$(-50,-100,-50)$ & 99979 & 99648 & 99593.5 & 99786.4 & 99784.6 & 99703.18 \\
\hline$(-50,-150,-50)$ & 99982.7 & 99649.8 & 99596.3 & 99783.4 & 99782.7 & 99703.05 \\
\hline$(-100,0,-50)$ & 99983.9 & 99646.1 & 99599.1 & 99786.1 & 99785.1 & 99704.1 \\
\hline$(-100,-50,-50)$ & 99980.3 & 99647.3 & 99593 & 99785.2 & 99789.2 & 99703.78 \\
\hline$(-100,-100,-50)$ & 99977.4 & 99644 & 99594.6 & 99785.4 & 99784.6 & 99702.25 \\
\hline$(-100,-150,-50)$ & 99984.7 & 99646.6 & 99591.4 & 99783.5 & 99782.7 & 99701.05 \\
\hline$(-150,0,-50)$ & 99983.6 & 99647.3 & 99597.2 & 99786.2 & 99788.8 & 99704.88 \\
\hline$(-150,-50,-50)$ & 99982.2 & 99648.2 & 99593.5 & 99787.4 & 99782.7 & 99702.95 \\
\hline$(-150,-100,-50)$ & 99980.1 & 99646.3 & 99596.3 & 99788.9 & 99781.5 & 99703.25 \\
\hline$(-150,-150,-50)$ & 99979.6 & 99646.1 & 99595.2 & 99784.3 & 99785.3 & 99702.73 \\
\hline$(50,0,100)$ & 99979.3 & 99647.3 & 99593.7 & 99783 & 99785.7 & 99702.43 \\
\hline$(-50,50,100)$ & 99981.2 & 99648.2 & 99599.1 & 99788.2 & 99787.2 & 99705.68 \\
\hline$(-50,100,100)$ & 99980.5 & 99646.3 & 99593.7 & 99788.6 & 99785.7 & 99703.58 \\
\hline$(50,150,100)$ & 99982.3 & 99648.5 & 99594.1 & 99786.4 & 99782.8 & 99702.95 \\
\hline$(100,0,100)$ & 99984.7 & 99644.2 & 99599.4 & 99783.4 & 99786.5 & 99703.38 \\
\hline$(100,50,100)$ & 99982.3 & 99648.2 & 99597.3 & 99786.1 & 99783.7 & 99703.83 \\
\hline$(100,100,100)$ & 99983.7 & 99649.8 & 99596.7 & 99785.2 & 99789.2 & 99705.23 \\
\hline$(100,150,100)$ & 99978.6 & 99648.2 & 99594.3 & 99785.4 & 99787.6 & 99703.88 \\
\hline$(150,0,100)$ & 99982.6 & 99646.3 & 99597.6 & 99783.5 & 99781.9 & 99702.33 \\
\hline$(150,50,100)$ & 99978.1 & 99648.5 & 99593.6 & 99786.2 & 99784.5 & 99703.2 \\
\hline$(150,100,100)$ & 99983.7 & 99644.2 & 99595.2 & 99787.4 & 99786.3 & 99703.28 \\
\hline$(150,150,100)$ & 99981.5 & 99648.2 & 99593.7 & 99788.9 & 99789.8 & 99705.15 \\
\hline$(-50,0,-100)$ & 99980.7 & 99649.8 & 99597.6 & 99785.3 & 99787.5 & 99705.05 \\
\hline$(-50,-50,-100)$ & 99981.3 & 99647.1 & 99593.6 & 99789.1 & 99782.7 & 99703.13 \\
\hline$(-50,-100,-100)$ & 99982.5 & 99646.6 & 99595.2 & 99788.2 & 99788.8 & 99704.7 \\
\hline$(-50,-150,-100)$ & 99980.9 & 99644.7 & 99593.7 & 99788.6 & 99782.7 & 99702.43 \\
\hline$(-100,0,-100)$ & 99981.3 & 99647.6 & 99599.1 & 99786.4 & 99781.5 & 99703.65 \\
\hline$(-100,-50,-100)$ & 99980.7 & 99642.3 & 99593.4 & 99783.4 & 99785.3 & 99701.1 \\
\hline$(-100,-100,-100)$ & 99980 & 99646.1 & 99594.6 & 99787.4 & 99785.7 & 99703.45 \\
\hline$(-100,-150,-100)$ & 99982.7 & 99647.3 & 99591.4 & 99788.9 & 99787.2 & 99703.7 \\
\hline$(-150,0,-100)$ & 99983.7 & 99644.4 & 99597 & 99785.3 & 99785.7 & 99703.15 \\
\hline$(-150,-50,-100)$ & 99980.3 & 99647 & 99593.5 & 99789.1 & 99782.8 & 99703 \\
\hline$(-150,-100,-100)$ & 99977.4 & 99647.3 & 99596.3 & 99788.2 & 99786.5 & 99704.58 \\
\hline$(-150,-150,-100)$ & 99984.8 & 99646.1 & 99593.7 & 99788.6 & 99783.7 & 99703.03 \\
\hline$(50,0,150)$ & 99982.9 & 99647.7 & 99591.4 & 99786 & 99789.2 & 99703.68 \\
\hline$(-50,50,150)$ & 99979.7 & 99648.5 & 99597.2 & 99783.4 & 99787.6 & 99704.18 \\
\hline$(-50,100,150)$ & 99981.3 & 99644.2 & 99593.5 & 99788.9 & 99782.7 & 99702.33 \\
\hline
\end{tabular}




\begin{tabular}{ccccccc}
$(50,150,150)$ & 99983.7 & 99648.2 & 99596.3 & 99783.9 & 99781.5 & 99702.48 \\
$(100,0,150)$ & 99984.7 & 99649.8 & 99599.1 & 99785.4 & 99785.3 & 99704.9 \\
$(100,50,150)$ & 99982 & 99647.1 & 99593.4 & 99783.5 & 99785.7 & 99702.43 \\
$(100,100,150)$ & 99983.7 & 99646.6 & 99594.6 & 99786.2 & 99787.2 & 99703.65 \\
$(100,150,150)$ & 99978.6 & 99644.7 & 99591 & 99787.4 & 99785.7 & 99702.3 \\
$(150,0,150)$ & 99985.2 & 99647.6 & 99597.2 & 99788.9 & 99783 & 99704.13 \\
$(150,50,150)$ & 99982.6 & 99642.3 & 99593.5 & 99785 & 99786.5 & 99701.9 \\
$(150,100,150)$ & 99978.1 & 99646.1 & 99596.3 & 99789.1 & 99783.7 & 99703.8 \\
$(150,150,150)$ & 99983.7 & 99647.3 & 99595.2 & 99788.2 & 99789.2 & 99704.98 \\
$(-50,0,-150)$ & 99981.5 & 99648.2 & 99597.2 & 99788.6 & 99782.7 & 99704.18 \\
$(-50,-50,-150)$ & 99977.6 & 99649.8 & 99594 & 99786.4 & 99788.8 & 99704.63 \\
$(-50,-100,-150)$ & 99983.6 & 99646 & 99596.3 & 99783.4 & 99782.7 & 99702.13 \\
$(-50,-150,-150)$ & 99981.7 & 99647.3 & 99595.2 & 99786 & 99781.5 & 99702.53 \\
$(-100,0,-150)$ & 99983.9 & 99644.4 & 99593.7 & 99785.2 & 99785.3 & 99702.15 \\
$(-100,-50,-150)$ & 99979.3 & 99646.6 & 99599.1 & 99785.4 & 99785.7 & 99704.2 \\
$(-100,-100,-150)$ & 99984.3 & 99647 & 99593.4 & 99786.4 & 99787.2 & 99703.58 \\
$(-100,-150,-150)$ & 99980.7 & 99648.2 & 99595 & 99783 & 99785.7 & 99702.98 \\
$(-150,0,-150)$ & 99981.3 & 99646.3 & 99591.4 & 99787.4 & 99782.8 & 99701.98 \\
$(-150,-50,-150)$ & 99983 & 99646.1 & 99597.2 & 99788.9 & 99786.5 & 99705.55 \\
$(-150,-100,-150)$ & 99980.9 & 99647.3 & 99593.5 & 99785.3 & 99783.7 & 99705.55 \\
$(-150,-150,-150)$ & 99978.4 & 99648 & 99596.3 & 99789 & 99789.2 & 99705.55 \\
\hline
\end{tabular}

Table 3

Pressure coefficient values for probe A at $34.4 \mathrm{~m} / \mathrm{s}$

\begin{tabular}{lllllll}
\hline Coordinate & $P_{\text {total (pitot) }}$ & $P_{\text {static }}$ pitot) & $C_{P_{\text {yaw }}}$ & $C_{P_{\text {pitch }}}$ & $C_{P_{\text {total }}}$ & $C_{P_{\text {static }}}$ \\
\hline$(0,0,0)$ & 107185.2 & 99829.3 & 0.187941 & 0.007605 & -26.0859 & -0.44849 \\
$(50,0,0)$ & 107183.4 & 99825.7 & 0.194901 & 0.007594 & -26.0495 & -0.44422 \\
$(50,50,0)$ & 107187.6 & 99827.3 & 0.191543 & 0.006505 & -26.047 & -0.44669 \\
$(50,100,0)$ & 107184.6 & 99822.8 & 0.167348 & -0.00533 & -25.5815 & -0.42476 \\
$(50,150,0)$ & 107183.6 & 99823.7 & 0.185528 & 0.023371 & -25.8923 & -0.42975 \\
$(100,0,0)$ & 107184.7 & 99829.3 & 0.204589 & 0.002539 & -26.1398 & -0.45842 \\
$(100,50,0)$ & 107184.6 & 99830.2 & 0.188111 & 0.00317 & -25.3612 & -0.45399 \\
$(100,100,0)$ & 107185.0 & 99831.5 & 0.17599 & 0.018317 & -25.8684 & -0.4573 \\
$(100,150,0)$ & 107183.4 & 99828.7 & 0.183633 & -0.02177 & -26.1486 & -0.45781 \\
$(150,0,0)$ & 107184.3 & 99833.9 & 0.172822 & -0.00143 & -25.8257 & -0.47078 \\
$(150,50,0)$ & 107188.7 & 99831.6 & 0.163162 & 0.010877 & -26.1461 & -0.47063 \\
$(150,100,0)$ & 107186.6 & 99260.3 & 0.176934 & -0.01218 & -25.7987 & 1.590616 \\
$(150,150,0)$ & 107184.6 & 99832.8 & 0.191025 & -0.0036 & -25.9611 & -0.46333
\end{tabular}




\begin{tabular}{|c|c|c|c|c|c|c|}
\hline$(-50,0,0)$ & 107182.0 & 99829.1 & 0.16906 & -0.01048 & -26.0132 & -0.45164 \\
\hline$(-50,-50,0)$ & 107187.1 & 99826.9 & 0.191994 & -0.01304 & -26.1137 & -0.45119 \\
\hline$(-50,-100,0)$ & 107184.6 & 99834.3 & 0.187275 & 0.011143 & -25.8925 & -0.4716 \\
\hline$(-50,-150,0)$ & 107183.6 & 99829.3 & 0.188286 & -0.00862 & -25.8818 & -0.45598 \\
\hline$(-100,0,0)$ & 107184.7 & 99826.9 & 0.175198 & 0.01442 & -25.9625 & -0.43836 \\
\hline$(-100,-50,0)$ & 107184.6 & 99829.4 & 0.180187 & 0.007897 & -25.8561 & -0.45549 \\
\hline$(-100,-100,0)$ & 107183.4 & 99831.5 & 0.183708 & -0.01563 & -25.5829 & -0.45918 \\
\hline$(-100,-150,0)$ & 107184.3 & 99828.7 & 0.193375 & 0.00573 & -25.7977 & -0.45712 \\
\hline$(-150,0,0)$ & 107188.7 & 99833.9 & 0.163714 & 0.009922 & -25.5276 & -0.46527 \\
\hline$(-150,-50,0)$ & 107186.6 & 99831.6 & 0.195148 & 0.012986 & -25.9901 & -0.45928 \\
\hline$(-150,-100,0)$ & 107184.6 & 99260.3 & 0.184861 & 0.001256 & -25.849 & 1.595594 \\
\hline$(-150,-150,0)$ & 107184.6 & 99832.8 & 0.183236 & -0.02152 & -25.8266 & -0.46428 \\
\hline$(50,0,50)$ & 107185.2 & 99834.3 & 0.188428 & 0.002489 & -25.6032 & -0.46885 \\
\hline$(50,50,50)$ & 107183.4 & 99833.9 & 0.169312 & 0.003228 & -25.8343 & -0.4709 \\
\hline$(50,100,50)$ & 107184.3 & 99832.8 & 0.169044 & 0.004993 & -25.6819 & -0.46398 \\
\hline$(50,150,50)$ & 107188.7 & 99832.8 & 0.186344 & 0.00183 & -26.3958 & -0.46586 \\
\hline$(100,0,50)$ & 107186.6 & 99831.6 & 0.170651 & -0.00249 & -25.6109 & -0.46209 \\
\hline$(100,50,50)$ & 107184.6 & 99831.5 & 0.176798 & -0.02333 & -26.2696 & -0.46523 \\
\hline$(100,100,50)$ & 107185.2 & 99829.4 & 0.191786 & 0.0175 & -25.7208 & -0.45 \\
\hline$(100,150,50)$ & 107185.2 & 99829.3 & 0.175354 & 0.003208 & -25.6745 & -0.45719 \\
\hline$(150,0,50)$ & 107185.2 & 99829.1 & 0.187705 & -0.00781 & -25.555 & -0.45321 \\
\hline$(150,50,50)$ & 107185.2 & 99828.7 & 0.174638 & -0.01232 & -26.1019 & -0.44783 \\
\hline$(150,100,50)$ & 107184.6 & 99826.9 & 0.195627 & 0.007497 & -25.706 & -0.44025 \\
\hline$(150,150,50)$ & 107183.4 & 99826.9 & 0.187642 & -0.01016 & -26.1456 & -0.44579 \\
\hline$(-50,0,-50)$ & 107184.3 & 99260.3 & 0.205544 & -0.01116 & -25.9294 & 1.594672 \\
\hline$(-50,-50,-50)$ & 107188.7 & 99834.3 & 0.170413 & 0.002175 & -26.1352 & -0.46954 \\
\hline$(-50,-100,-50)$ & 107186.6 & 99834.3 & 0.198027 & -0.00652 & -26.0918 & -0.4747 \\
\hline$(-50,-150,-50)$ & 107184.6 & 99833.9 & 0.191311 & -0.0025 & -25.7533 & -0.46791 \\
\hline$(-100,0,-50)$ & 107184.6 & 99833.9 & 0.167977 & -0.00357 & -25.7352 & -0.4639 \\
\hline$(-100,-50,-50)$ & 107185.2 & 99832.8 & 0.194919 & 0.014465 & -26.0552 & -0.46659 \\
\hline$(-100,-100,-50)$ & 107183.4 & 99832.8 & 0.180992 & -0.00291 & -26.1892 & -0.47447 \\
\hline$(-100,-150,-50)$ & 107184.3 & 99832.8 & 0.194606 & -0.00282 & -25.382 & -0.46448 \\
\hline$(-150,0,-50)$ & 107188.7 & 99831.6 & 0.179747 & 0.009328 & -25.8502 & -0.45466 \\
\hline$(-150,-50,-50)$ & 107185.2 & 99831.6 & 0.195882 & -0.01683 & -25.7942 & -0.4607 \\
\hline$(-150,-100,-50)$ & 107184.6 & 99831.5 & 0.180603 & -0.02673 & -26.0231 & -0.46325 \\
\hline$(-150,-150,-50)$ & 107183.4 & 99825.7 & 0.183837 & 0.003612 & -26.0181 & -0.44415 \\
\hline$(50,0,100)$ & 107184.3 & 99827.3 & 0.193589 & 0.009752 & -26.0226 & -0.45102 \\
\hline$(-50,50,100)$ & 107188.7 & 99822.8 & 0.178205 & -0.00363 & -26.1592 & -0.4251 \\
\hline$(-50,100,100)$ & 107186.6 & 99823.7 & 0.189943 & -0.01047 & -26.0218 & -0.43378 \\
\hline$(50,150,100)$ & 107184.6 & 99829.3 & 0.194738 & -0.01289 & -25.7824 & -0.4523 \\
\hline$(100,0,100)$ & 107184.6 & 99830.2 & 0.159246 & 0.011019 & -25.5928 & -0.45081 \\
\hline
\end{tabular}




\begin{tabular}{|c|c|c|c|c|c|c|}
\hline$(100,50,100)$ & 107185.2 & 99831.5 & 0.182781 & -0.00862 & -25.8656 & -0.45848 \\
\hline$(100,100,100)$ & 107183.4 & 99828.7 & 0.190681 & 0.014364 & -25.8539 & -0.4434 \\
\hline$(100,150,100)$ & 107184.3 & 99833.9 & 0.196196 & 0.008008 & -26.2288 & -0.47329 \\
\hline$(150,0,100)$ & 107188.7 & 99831.6 & 0.173758 & -0.00571 & -25.7108 & -0.46124 \\
\hline$(150,50,100)$ & 107184.3 & 99260.3 & 0.199709 & -0.00618 & -26.2139 & 1.611131 \\
\hline$(150,100,100)$ & 107188.7 & 99832.8 & 0.174735 & -0.00392 & -25.6931 & -0.46189 \\
\hline$(150,150,100)$ & 107186.6 & 99829.1 & 0.197214 & 0.003257 & -26.0724 & -0.44853 \\
\hline$(-50,0,-100)$ & 107184.6 & 99826.9 & 0.189371 & 0.007981 & -26.1342 & -0.44205 \\
\hline$(-50,-50,-100)$ & 107184.6 & 99834.3 & 0.192325 & -0.02301 & -25.8949 & -0.47156 \\
\hline$(-50,-100,-100)$ & 107185.2 & 99829.3 & 0.185025 & 0.00216 & -25.9277 & -0.44852 \\
\hline$(-50,-150,-100)$ & 107183.4 & 99826.9 & 0.18314 & -0.02119 & -25.8639 & -0.44699 \\
\hline$(-100,0,-100)$ & 107184.3 & 99829.4 & 0.17468 & -0.01765 & -25.9427 & -0.45291 \\
\hline$(-100,-50,-100)$ & 107188.7 & 99831.5 & 0.174893 & 0.006795 & -25.7797 & -0.46638 \\
\hline$(-100,-100,-100)$ & 107185.2 & 99828.7 & 0.186493 & -0.00616 & -26.0932 & -0.45356 \\
\hline$(-100,-150,-100)$ & 107188.7 & 99833.9 & 0.200358 & -0.00609 & -25.828 & -0.46667 \\
\hline$(-150,0,-100)$ & 107185.2 & 99826.9 & 0.168241 & 0.001426 & -25.6693 & -0.4411 \\
\hline$(-150,-50,-100)$ & 107184.6 & 99832.8 & 0.191489 & -0.02272 & -25.9802 & -0.46809 \\
\hline$(-150,-100,-100)$ & 107183.4 & 99832.8 & 0.186933 & -0.00623 & -26.4124 & -0.46999 \\
\hline$(-150,-150,-100)$ & 107184.3 & 99831.6 & 0.185964 & -0.01739 & -25.5505 & -0.4563 \\
\hline$(50,0,150)$ & 107188.7 & 99831.5 & 0.20163 & 0.010028 & -25.8064 & -0.45778 \\
\hline$(-50,50,150)$ & 107186.6 & 99829.4 & 0.18619 & 0.015244 & -26.157 & -0.4545 \\
\hline$(-50,100,150)$ & 107184.6 & 99829.4 & 0.181737 & -0.02222 & -25.8206 & -0.45551 \\
\hline$(50,150,150)$ & 107184.6 & 99829.3 & 0.18455 & -0.00853 & -25.6055 & -0.45097 \\
\hline$(100,0,150)$ & 107185.2 & 99825.7 & 0.181201 & -0.00036 & -25.7345 & -0.43174 \\
\hline$(100,50,150)$ & 107183.4 & 99827.3 & 0.191871 & 0.007861 & -25.7296 & -0.44618 \\
\hline$(100,100,150)$ & 107184.3 & 99822.8 & 0.185681 & 0.003571 & -25.7118 & -0.42546 \\
\hline$(100,150,150)$ & 107188.7 & 99823.7 & 0.192906 & -0.00615 & -26.0952 & -0.43938 \\
\hline$(150,0,150)$ & 107185.2 & 99829.3 & 0.179312 & -0.0217 & -25.616 & -0.44534 \\
\hline$(150,50,150)$ & 107184.3 & 99830.2 & 0.173851 & 0.004275 & -25.6562 & -0.45707 \\
\hline$(150,100,150)$ & 107185.2 & 99831.5 & 0.181553 & -0.01969 & -26.2746 & -0.46555 \\
\hline$(150,150,150)$ & 107185.2 & 99828.7 & 0.186923 & 0.003588 & -25.8374 & -0.4439 \\
\hline$(-50,0,-150)$ & 107188.7 & 99833.9 & 0.1839 & -0.02127 & -25.9883 & -0.46777 \\
\hline$(-50,-50,-150)$ & 107185.2 & 99831.6 & 0.206246 & 0.008792 & -26.404 & -0.46515 \\
\hline$(-50,-100,-150)$ & 107184.6 & 99260.3 & 0.176925 & -0.00249 & -25.5831 & 1.569678 \\
\hline$(-50,-150,-150)$ & 107183.4 & 99832.8 & 0.186621 & -0.01648 & -25.7962 & -0.46664 \\
\hline$(-100,0,-150)$ & 107184.3 & 99829.1 & 0.179947 & 0.000355 & -25.556 & -0.45058 \\
\hline$(-100,-50,-150)$ & 107188.7 & 99826.9 & 0.172664 & 0.001091 & -26.2065 & -0.44602 \\
\hline$(-100,-100,-150)$ & 107186.6 & 99834.3 & 0.192003 & 0.00285 & -25.6561 & -0.46567 \\
\hline$(-100,-150,-150)$ & 107184.6 & 99829.3 & 0.192997 & 0.008282 & -25.939 & -0.45486 \\
\hline$(-150,0,-150)$ & 107184.6 & 99826.9 & 0.196545 & -0.01647 & -25.7882 & -0.44724 \\
\hline$(-150,-50,-150)$ & 107185.2 & 99829.4 & 0.188015 & 0.000367 & -26.4097 & -0.45135 \\
\hline
\end{tabular}




\begin{tabular}{lllllll}
$(-150,-100,-150)$ & 107183.4 & 99831.5 & 0.188015 & 0.000367 & -26.4097 & -0.45135 \\
$(-150,-150,-150)$ & 107184.3 & 99828.7 & 0.188015 & 0.000367 & -26.4097 & -0.45135 \\
\hline
\end{tabular}

\section{Table 4}

Pressure values for probe B at $34.4 \mathrm{~m} / \mathrm{s}$

\begin{tabular}{|c|c|c|c|c|c|c|}
\hline Coordinate & $P_{6}$ & $P_{7}$ & $P_{8}$ & $P_{9}$ & $P_{10}$ & $\bar{P}$ \\
\hline$(0,0,0)$ & 99980.9 & 99782.7 & 99703.0 & 99648.5 & 99644.2 & 99694.59 \\
\hline$(50,0,0)$ & 99978.4 & 99781.5 & 99703.4 & 99644.2 & 99648 & 99694.32 \\
\hline$(50,50,0)$ & 99981.3 & 99785.3 & 99703.8 & 99648 & 99649.8 & 99696.78 \\
\hline$(50,100,0)$ & 99980.7 & 99785.7 & 99705.2 & 99649.8 & 99646.1 & 99696.71 \\
\hline$(50,150,0)$ & 99982.7 & 99787.2 & 99703.9 & 99646.1 & 99647.3 & 99696.12 \\
\hline$(100,0,0)$ & 99981.1 & 99785.7 & 99702.3 & 99647.3 & 99644 & 99694.93 \\
\hline$(100,50,0)$ & 99983.7 & 99782.8 & 99703.2 & 99644 & 99646.6 & 99694.25 \\
\hline$(100,100,0)$ & 99980 & 99786.5 & 99703.3 & 99646.6 & 99647.3 & 99695.92 \\
\hline$(100,150,0)$ & 99984.8 & 99783.7 & 99705.2 & 99647.3 & 99648.2 & 99696.09 \\
\hline$(150,0,0)$ & 99981.5 & 99789.2 & 99705.1 & 99648.2 & 99646.3 & 99697.19 \\
\hline$(150,50,0)$ & 99983.4 & 99787.6 & 99703.1 & 99646.3 & 99646.1 & 99695.78 \\
\hline$(150,100,0)$ & 99982 & 99781.9 & 99704.7 & 99646.1 & 99647.3 & 99695 \\
\hline$(150,150,0)$ & 99983.7 & 99784.5 & 99702.4 & 99647.3 & 99648.2 & 99695.61 \\
\hline$(-50,0,0)$ & 99981.4 & 99786.3 & 99703.7 & 99648.2 & 99646.3 & 99696.11 \\
\hline$(-50,-50,0)$ & 99983.1 & 99789.8 & 99701.1 & 99646.3 & 99648.5 & 99696.43 \\
\hline$(-50,-100,0)$ & 99978.7 & 99787.75 & 99703.5 & 99648.5 & 99644.2 & 99695.98 \\
\hline$(-50,-150,0)$ & 99982.9 & 99782.9 & 99703.7 & 99644.2 & 99647.8 & 99694.65 \\
\hline$(-100,0,0)$ & 99978.2 & 99784.6 & 99703.2 & 99648.2 & 99646.3 & 99695.56 \\
\hline$(-100,-50,0)$ & 99983.4 & 99782.7 & 99703.0 & 99649.8 & 99647.1 & 99695.65 \\
\hline$(-100,-100,0)$ & 99981.6 & 99785.1 & 99704.6 & 99648.2 & 99646.6 & 99696.12 \\
\hline$(-100,-150,0)$ & 99983.2 & 99789.7 & 99703.0 & 99646.3 & 99647.3 & 99696.58 \\
\hline$(-150,0,0)$ & 99981 & 99787.4 & 99703.7 & 99648.5 & 99648.2 & 99696.94 \\
\hline$(-150,-50,0)$ & 99983.7 & 99786.3 & 99704.2 & 99644.2 & 99646.3 & 99695.24 \\
\hline$(-150,-100,0)$ & 99979.6 & 99785.1 & 99702.3 & 99648.2 & 99646.1 & 99695.43 \\
\hline$(-150,-150,0)$ & 99981.1 & 99789.2 & 99702.5 & 99649.8 & 99647.3 & 99697.19 \\
\hline$(50,0,50)$ & 99980.6 & 99784.6 & 99704.9 & 99647.1 & 99648.2 & 99696.2 \\
\hline$(50,50,50)$ & 99979 & 99782.7 & 99702.4 & 99646.6 & 99646.3 & 99694.51 \\
\hline$(50,100,50)$ & 99982.7 & 99788.8 & 99703.5 & 99644.7 & 99648.5 & 99696.36 \\
\hline$(50,150,50)$ & 99983.9 & 99782.7 & 99703.7 & 99647.6 & 99644.2 & 99694.55 \\
\hline$(100,0,50)$ & 99980.3 & 99781.5 & 99703.2 & 99642.3 & 99647.8 & 99693.69 \\
\hline$(100,50,50)$ & 99977.4 & 99785.3 & 99703.0 & 99646.1 & 99646.3 & 99695.18 \\
\hline$(100,100,50)$ & 99983.6 & 99785.7 & 99704.6 & 99647.3 & 99647.1 & 99696.17 \\
\hline
\end{tabular}




\begin{tabular}{|c|c|c|c|c|c|c|}
\hline$(100,150,50)$ & 99982.2 & 99787.2 & 99703.0 & 99644.4 & 99646.6 & 99695.31 \\
\hline$(150,0,50)$ & 99980.1 & 99785.7 & 99703.7 & 99646.1 & 99644.7 & 99695.04 \\
\hline$(150,50,50)$ & 99979.6 & 99782.8 & 99704.2 & 99647.3 & 99647.6 & 99695.47 \\
\hline$(150,100,50)$ & 99979.3 & 99786.5 & 99702.3 & 99648.2 & 99642.3 & 99694.83 \\
\hline$(150,150,50)$ & 99981.2 & 99783.7 & 99702.5 & 99646.3 & 99646.1 & 99694.64 \\
\hline$(-50,0,-50)$ & 99980.5 & 99789.2 & 99704.9 & 99648.5 & 99647.3 & 99697.48 \\
\hline$(-50,-50,-50)$ & 99982.3 & 99787.6 & 99702.4 & 99644.2 & 99644.4 & 99694.66 \\
\hline$(-50,-100,-50)$ & 99984.7 & 99781.9 & 99703.7 & 99648.2 & 99646.6 & 99695.09 \\
\hline$(-50,-150,-50)$ & 99982.3 & 99784.5 & 99702.3 & 99649.8 & 99647.3 & 99695.98 \\
\hline$(-100,0,-50)$ & 99983.7 & 99786.3 & 99704.1 & 99648.2 & 99645.2 & 99695.96 \\
\hline$(-100,-50,-50)$ & 99978.6 & 99789.8 & 99701.9 & 99648.7 & 99646.3 & 99696.68 \\
\hline$(-100,-100,-50)$ & 99982.6 & 99787.75 & 99703.8 & 99647.5 & 99648.5 & 99696.89 \\
\hline$(-100,-150,-50)$ & 99978.1 & 99782.9 & 99705.0 & 99646.1 & 99644.2 & 99694.54 \\
\hline$(-150,0,-50)$ & 99983.7 & 99784.6 & 99704.2 & 99647.7 & 99648 & 99696.17 \\
\hline$(-150,-50,-50)$ & 99981.5 & 99782.7 & 99704.6 & 99648.5 & 99649.8 & 99696.41 \\
\hline$(-150,-100,-50)$ & 99980.7 & 99787.2 & 99702.1 & 99644.2 & 99646.1 & 99694.91 \\
\hline$(-150,-150,-50)$ & 99981.3 & 99785.7 & 99702.5 & 99648.2 & 99647.3 & 99695.93 \\
\hline$(50,0,100)$ & 99982.5 & 99783 & 99702.2 & 99649.8 & 99644 & 99694.79 \\
\hline$(-50,50,100)$ & 99980.9 & 99786.5 & 99704.2 & 99647.1 & 99646.6 & 99696.1 \\
\hline$(-50,100,100)$ & 99981.3 & 99783.7 & 99703.6 & 99646.6 & 99647.3 & 99695.29 \\
\hline$(50,150,100)$ & 99980.7 & 99789.2 & 99703.0 & 99644.7 & 99648.2 & 99696.27 \\
\hline$(100,0,100)$ & 99980 & 99782.7 & 99702.0 & 99647.6 & 99646.3 & 99694.64 \\
\hline$(100,50,100)$ & 99982.7 & 99788.8 & 99705.6 & 99642.3 & 99646.1 & 99695.69 \\
\hline$(100,100,100)$ & 99983.7 & 99782.7 & 99705.6 & 99646.1 & 99647.3 & 99695.41 \\
\hline$(100,150,100)$ & 99980.3 & 99781.5 & 99705.6 & 99647.3 & 99648.2 & 99695.64 \\
\hline$(150,0,100)$ & 99977.4 & 99785.3 & 99702.9 & 99648.2 & 99646.3 & 99695.66 \\
\hline$(150,50,100)$ & 99984.8 & 99785.7 & 99703.7 & 99649.8 & 99648.5 & 99696.93 \\
\hline$(150,100,100)$ & 99982.9 & 99787.2 & 99703.3 & 99646 & 99644.2 & 99695.19 \\
\hline$(150,150,100)$ & 99979.7 & 99785.7 & 99704.2 & 99647.3 & 99648.2 & 99696.34 \\
\hline$(-50,0,-100)$ & 99981.3 & 99782.8 & 99702.9 & 99644.4 & 99649.8 & 99694.98 \\
\hline$(-50,-50,-100)$ & 99983.7 & 99786.5 & 99701.3 & 99646.6 & 99648.2 & 99695.66 \\
\hline$(-50,-100,-100)$ & 99984.7 & 99783.7 & 99704.2 & 99647 & 99646.3 & 99695.37 \\
\hline$(-50,-150,-100)$ & 99982 & 99789.2 & 99702.6 & 99648.2 & 99648.5 & 99697.11 \\
\hline$(-100,0,-100)$ & 99983.7 & 99784.5 & 99702.6 & 99646 & 99644.2 & 99694.35 \\
\hline$(-100,-50,-100)$ & 99978.6 & 99786.3 & 99701.8 & 99647.3 & 99648.2 & 99695.9 \\
\hline$(-100,-100,-100)$ & 99985.2 & 99789.8 & 99704.4 & 99644.4 & 99649.8 & 99697.1 \\
\hline$(-100,-150,-100)$ & 99982.6 & 99787.75 & 99704.3 & 99646.6 & 99647.1 & 99696.43 \\
\hline$(-150,0,-100)$ & 99978.1 & 99782.9 & 99704.1 & 99647 & 99646.6 & 99695.22 \\
\hline$(-150,-50,-100)$ & 99983.7 & 99784.6 & 99702.4 & 99648.2 & 99644.7 & 99694.96 \\
\hline$(-150,-100,-100)$ & 99981.5 & 99782.7 & 99703.1 & 99646.3 & 99647.6 & 99694.93 \\
\hline$(-150,-150,-100)$ & 99977.6 & 99787.2 & 99702.4 & 99647.3 & 99642.3 & 99694.8 \\
\hline
\end{tabular}




\begin{tabular}{|c|c|c|c|c|c|c|}
\hline$(50,0,150)$ & 99983.6 & 99785.7 & 99705.3 & 99648.2 & 99646.1 & 99696.33 \\
\hline$(-50,50,150)$ & 99981.7 & 99783 & 99702.5 & 99649.8 & 99647.3 & 99695.6 \\
\hline$(-50,100,150)$ & 99983.9 & 99786.5 & 99702.3 & 99646 & 99644.4 & 99694.82 \\
\hline$(50,150,150)$ & 99979.3 & 99783.7 & 99701.1 & 99647.3 & 99647 & 99694.66 \\
\hline$(100,0,150)$ & 99984.3 & 99789.2 & 99702.6 & 99644.4 & 99647.3 & 99695.88 \\
\hline$(100,50,150)$ & 99980.7 & 99782.7 & 99704.3 & 99646.6 & 99646.1 & 99694.92 \\
\hline$(100,100,150)$ & 99981.3 & 99788.8 & 99704.8 & 99649 & 99647.3 & 99697.55 \\
\hline$(100,150,150)$ & 99983 & 99782.7 & 99703.3 & 99649.8 & 99648.2 & 99696.01 \\
\hline$(150,0,150)$ & 99980.9 & 99781.5 & 99704.3 & 99646 & 99649.8 & 99695.41 \\
\hline$(150,50,150)$ & 99978.4 & 99785.3 & 99704.1 & 99647.3 & 99646 & 99695.69 \\
\hline$(150,100,150)$ & 99984.7 & 99785.7 & 99702.4 & 99644.4 & 99647.3 & 99694.94 \\
\hline$(150,150,150)$ & 99982 & 99787.2 & 99703.1 & 99646.6 & 99644.4 & 99695.33 \\
\hline$(-50,0,-150)$ & 99983.7 & 99785.7 & 99702.4 & 99647 & 99646.6 & 99695.5 \\
\hline$(-50,-50,-150)$ & 99978.6 & 99782.8 & 99705.3 & 99648.2 & 99647 & 99695.9 \\
\hline$(-50,-100,-150)$ & 99985.2 & 99784.5 & 99702.5 & 99646 & 99648.2 & 99695.33 \\
\hline$(-50,-150,-150)$ & 99982.6 & 99786.3 & 99702.3 & 99647.3 & 99646.3 & 99695.54 \\
\hline$(-100,0,-150)$ & 99978.1 & 99789.8 & 99701.1 & 99647.4 & 99644.4 & 99695.66 \\
\hline$(-100,-50,-150)$ & 99983.7 & 99787.75 & 99702.6 & 99643.6 & 99646.6 & 99695.14 \\
\hline$(-100,-100,-150)$ & 99981.5 & 99782.9 & 99704.3 & 99647.3 & 99649 & 99695.94 \\
\hline$(-100,-150,-150)$ & 99977.6 & 99784.6 & 99704.8 & 99648.2 & 99649.8 & 99696.85 \\
\hline$(-150,0,-150)$ & 99983.6 & 99782.7 & 99703.3 & 99646.3 & 99646 & 99694.61 \\
\hline$(-150,-50,-150)$ & 99981.7 & 99787.2 & 99702.4 & 99645.3 & 99647.3 & 99695.56 \\
\hline$(-150,-100,-150)$ & 99983.9 & 99785.7 & 99702.6 & 99648.2 & 99644.4 & 99695.23 \\
\hline$(-150,-150,-150)$ & 99979.3 & 99783 & 99702.7 & 99649.8 & 99646.6 & 99695.48 \\
\hline
\end{tabular}

Table 5

Pressure coefficient values for probe B at $34.4 \mathrm{~m} / \mathrm{s}$

\begin{tabular}{lllllll}
\hline Coordinate & $P_{\text {total (pitot) }}$ & $P_{\text {Static(pitot) }}$ & $C_{P_{\text {yaw }}}$ & $C_{P_{\text {pitch }}}$ & $C_{P_{\text {total }}}$ & $C_{P_{\text {static }}}$ \\
\hline$(0,0,0)$ & 107185.2 & 99829.3 & 0.278542 & -0.01501 & -25.1624 & -0.47050 \\
$(50,0,0)$ & 107183.4 & 99825.7 & 0.275009 & 0.01408 & -25.3625 & -0.46248 \\
$(50,50,0)$ & 107187.6 & 99827.3 & 0.286361 & 0.005624 & -25.328 & -0.45874 \\
$(50,100,0)$ & 107184.6 & 99822.8 & 0.283369 & -0.01303 & -25.3664 & -0.444 \\
$(50,150,0)$ & 107183.6 & 99823.7 & 0.290755 & 0.004187 & -25.1269 & -0.44518 \\
$(100,0,0)$ & 107184.7 & 99829.3 & 0.291349 & -0.01013 & -25.1726 & -0.46954 \\
$(100,50,0)$ & 107184.6 & 99830.2 & 0.275004 & 0.007601 & -24.8779 & -0.46968 \\
$(100,100,0)$ & 107185.0 & 99831.5 & 0.292653 & 0.002461 & -25.3347 & -0.47676 \\
$(100,150,0)$ & 107183.4 & 99828.7 & 0.27207 & 0.003117 & -24.9333 & -0.45932 \\
$(150,0,0)$ & 107184.3 & 99833.9 & 0.295977 & -0.00668 & -25.3341 & -0.48085 \\
$(150,50,0)$ & 107188.7 & 99831.6 & 0.293705 & -0.0007 & -25.0516 & -0.47222
\end{tabular}




\begin{tabular}{|c|c|c|c|c|c|c|}
\hline$(150,100,0)$ & 107186.6 & 99260.3 & 0.268802 & 0.004178 & -25.085 & 1.513579 \\
\hline$(150,150,0)$ & 107184.6 & 99832.8 & 0.28489 & 0.003124 & -24.995 & -0.47621 \\
\hline$(-50,0,0)$ & 107182.0 & 99829.1 & 0.289708 & -0.00666 & -25.2398 & -0.46615 \\
\hline$(-50,-50,0)$ & 107187.1 & 99826.9 & 0.30941 & 0.007674 & -25.1295 & -0.45513 \\
\hline$(-50,-100,0)$ & 107184.6 & 99834.3 & 0.29817 & -0.01521 & -25.4873 & -0.48926 \\
\hline$(-50,-150,0)$ & 107183.6 & 99829.3 & 0.274761 & 0.012489 & -24.9807 & -0.46713 \\
\hline$(-100,0,0)$ & 107184.7 & 99826.9 & 0.288178 & -0.00672 & -25.4973 & -0.46469 \\
\hline$(-100,-50,0)$ & 107184.6 & 99829.4 & 0.276977 & -0.00938 & -25.0259 & -0.46481 \\
\hline$(-100,-100,0)$ & 107183.4 & 99831.5 & 0.282068 & -0.0056 & -25.2267 & -0.47422 \\
\hline$(-100,-150,0)$ & 107184.3 & 99828.7 & 0.302405 & 0.003489 & -25.1243 & -0.46096 \\
\hline$(-150,0,0)$ & 107188.7 & 99833.9 & 0.294644 & -0.00106 & -25.3649 & -0.48198 \\
\hline$(-150,-50,0)$ & 107186.6 & 99831.6 & 0.284705 & 0.00728 & -24.9705 & -0.47271 \\
\hline$(-150,-100,0)$ & 107184.6 & 99260.3 & 0.291288 & -0.00739 & -25.3547 & 1.531242 \\
\hline$(-150,-150,0)$ & 107184.6 & 99832.8 & 0.305471 & -0.00881 & -25.3728 & -0.47764 \\
\hline$(50,0,50)$ & 107185.2 & 99834.3 & 0.280239 & 0.003868 & -25.3327 & -0.48558 \\
\hline$(50,50,50)$ & 107183.4 & 99833.9 & 0.281772 & -0.00105 & -25.2865 & -0.48928 \\
\hline$(50,100,50)$ & 107184.3 & 99832.8 & 0.298075 & 0.013271 & -25.1507 & -0.47649 \\
\hline$(50,150,50)$ & 107188.7 & 99832.8 & 0.273026 & -0.01175 & -24.8999 & -0.4778 \\
\hline$(100,0,50)$ & 107186.6 & 99831.6 & 0.273366 & 0.01919 & -25.143 & -0.48118 \\
\hline$(100,50,50)$ & 107184.6 & 99831.5 & 0.291611 & 0.000709 & -25.5371 & -0.48304 \\
\hline$(100,100,50)$ & 107185.2 & 99829.4 & 0.282241 & -0.0007 & -25.0551 & -0.46352 \\
\hline$(100,150,50)$ & 107185.2 & 99829.3 & 0.293401 & 0.007668 & -25.1069 & -0.46705 \\
\hline$(150,0,50)$ & 107185.2 & 99829.1 & 0.28775 & -0.00491 & -25.2762 & -0.47028 \\
\hline$(150,50,50)$ & 107185.2 & 99828.7 & 0.276721 & 0.001056 & -25.3602 & -0.46891 \\
\hline$(150,100,50)$ & 107184.6 & 99826.9 & 0.295902 & -0.02074 & -25.329 & -0.46426 \\
\hline$(150,150,50)$ & 107183.4 & 99826.9 & 0.283452 & -0.0007 & -25.1335 & -0.46154 \\
\hline$(-50,0,-50)$ & 107184.3 & 99260.3 & 0.297854 & -0.00424 & -25.4529 & 1.544652 \\
\hline$(-50,-50,-50)$ & 107188.7 & 99834.3 & 0.296113 & 0.000695 & -25.0532 & -0.48547 \\
\hline$(-50,-100,-50)$ & 107186.6 & 99834.3 & 0.270189 & -0.00552 & -24.8674 & -0.48069 \\
\hline$(-50,-150,-50)$ & 107184.6 & 99833.9 & 0.287086 & -0.00873 & -25.1543 & -0.48171 \\
\hline$(-100,0,-50)$ & 107184.6 & 99833.9 & 0.285584 & -0.01043 & -25.0254 & -0.4794 \\
\hline$(-100,-50,-50)$ & 107185.2 & 99832.8 & 0.311785 & -0.00851 & -25.5622 & -0.48284 \\
\hline$(-100,-100,-50)$ & 107183.4 & 99832.8 & 0.293827 & 0.0035 & -25.2028 & -0.4757 \\
\hline$(-100,-150,-50)$ & 107184.3 & 99832.8 & 0.274813 & -0.0067 & -25.4137 & -0.48758 \\
\hline$(-150,0,-50)$ & 107188.7 & 99831.6 & 0.279709 & 0.001739 & -25.0581 & -0.47101 \\
\hline$(-150,-50,-50)$ & 107185.2 & 99831.6 & 0.273857 & 0.00456 & -25.2679 & -0.47421 \\
\hline$(-150,-100,-50)$ & 107184.6 & 99831.5 & 0.29768 & 0.006648 & -25.2066 & -0.47795 \\
\hline$(-150,-150,-50)$ & 107183.4 & 99825.7 & 0.291465 & -0.00315 & -25.2377 & -0.45474 \\
\hline$(50,0,100)$ & 107184.3 & 99827.3 & 0.280315 & -0.01877 & -25.0312 & -0.46057 \\
\hline$(-50,50,100)$ & 107188.7 & 99822.8 & 0.288975 & -0.00176 & -25.3083 & -0.44487 \\
\hline$(-50,100,100)$ & 107186.6 & 99823.7 & 0.280151 & 0.002447 & -25.1928 & -0.44896 \\
\hline
\end{tabular}




\begin{tabular}{|c|c|c|c|c|c|c|}
\hline$(50,150,100)$ & 107184.6 & 99829.3 & 0.303149 & 0.012305 & -25.3274 & -0.46771 \\
\hline$(100,0,100)$ & 107184.6 & 99830.2 & 0.283289 & -0.00456 & -25.2846 & -0.47571 \\
\hline$(100,50,100)$ & 107185.2 & 99831.5 & 0.290057 & 0.01324 & -25.0948 & -0.47319 \\
\hline$(100,100,100)$ & 107183.4 & 99828.7 & 0.267615 & 0.004163 & -24.9739 & -0.46234 \\
\hline$(100,150,100)$ & 107184.3 & 99833.9 & 0.266807 & 0.003162 & -25.3072 & -0.48571 \\
\hline$(150,0,100)$ & 107188.7 & 99831.6 & 0.292648 & -0.00674 & -25.5958 & -0.4825 \\
\hline$(150,50,100)$ & 107184.3 & 99260.3 & 0.284846 & -0.00452 & -25.0091 & 1.516717 \\
\hline$(150,100,100)$ & 107188.7 & 99832.8 & 0.291784 & -0.0066 & -25.0451 & -0.4783 \\
\hline$(150,150,100)$ & 107186.6 & 99829.1 & 0.287712 & 0.003176 & -25.4341 & -0.46851 \\
\hline$(-50,0,-100)$ & 107184.6 & 99826.9 & 0.278972 & 0.01886 & -25.1583 & -0.46074 \\
\hline$(-50,-50,-100)$ & 107184.6 & 99834.3 & 0.295702 & 0.005555 & -24.9993 & -0.48133 \\
\hline$(-50,-100,-100)$ & 107185.2 & 99829.3 & 0.274858 & -0.00346 & -24.8868 & -0.4629 \\
\hline$(-50,-150,-100)$ & 107183.4 & 99826.9 & 0.303835 & 0.001052 & -25.2503 & -0.4551 \\
\hline$(-100,0,-100)$ & 107184.3 & 99829.4 & 0.283048 & -0.00657 & -24.8854 & -0.46674 \\
\hline$(-100,-50,-100)$ & 107188.7 & 99831.5 & 0.298903 & 0.003184 & -25.5044 & -0.47966 \\
\hline$(-100,-100,-100)$ & 107185.2 & 99828.7 & 0.296425 & 0.018743 & -24.9914 & -0.45679 \\
\hline$(-100,-150,-100)$ & 107188.7 & 99833.9 & 0.29178 & 0.001747 & -25.1807 & -0.48039 \\
\hline$(-150,0,-100)$ & 107185.2 & 99826.9 & 0.27865 & -0.00247 & -25.4776 & -0.4655 \\
\hline$(-150,-50,-100)$ & 107184.6 & 99832.8 & 0.284861 & -0.01212 & -24.9393 & -0.47738 \\
\hline$(-150,-100,-100)$ & 107183.4 & 99832.8 & 0.277763 & 0.004536 & -25.1308 & -0.48111 \\
\hline$(-150,-150,-100)$ & 107184.3 & 99831.6 & 0.299859 & -0.01768 & -25.4834 & -0.48373 \\
\hline$(50,0,150)$ & 107188.7 & 99831.5 & 0.279871 & -0.00731 & -25.0808 & -0.47054 \\
\hline$(-50,50,150)$ & 107186.6 & 99829.4 & 0.280671 & -0.00874 & -25.1832 & -0.46767 \\
\hline$(-50,100,150)$ & 107184.6 & 99829.4 & 0.291354 & -0.00588 & -24.9089 & -0.46555 \\
\hline$(50,150,150)$ & 107184.6 & 99829.3 & 0.290369 & -0.00246 & -25.314 & -0.47301 \\
\hline$(100,0,150)$ & 107185.2 & 99825.7 & 0.300251 & 0.010055 & -24.9664 & -0.45012 \\
\hline$(100,50,150)$ & 107183.4 & 99827.3 & 0.274423 & -0.00175 & -25.2034 & -0.46323 \\
\hline$(100,100,150)$ & 107184.3 & 99822.8 & 0.295994 & -0.00705 & -25.3853 & -0.4414 \\
\hline$(100,150,150)$ & 107188.7 & 99823.7 & 0.277057 & -0.00558 & -25.1531 & -0.44571 \\
\hline$(150,0,150)$ & 107185.2 & 99829.3 & 0.27059 & 0.01296 & -25.2352 & -0.46898 \\
\hline$(150,50,150)$ & 107184.3 & 99830.2 & 0.287312 & -0.00424 & -25.489 & -0.47578 \\
\hline$(150,100,150)$ & 107185.2 & 99831.5 & 0.287649 & 0.010008 & -24.8498 & -0.47129 \\
\hline$(150,150,150)$ & 107185.2 & 99828.7 & 0.293057 & -0.00767 & -25.0995 & -0.46476 \\
\hline$(-50,0,-150)$ & 107188.7 & 99833.9 & 0.289035 & -0.00243 & -25 & -0.48022 \\
\hline$(-50,-50,-150)$ & 107185.2 & 99831.6 & 0.274142 & -0.00318 & -25.4921 & -0.48001 \\
\hline$(-50,-100,-150)$ & 107184.6 & 99260.3 & 0.282881 & 0.007245 & -24.8362 & 1.500733 \\
\hline$(-50,-150,-150)$ & 107183.4 & 99832.8 & 0.292713 & -0.00348 & -25.0848 & -0.47815 \\
\hline$(-100,0,-150)$ & 107184.3 & 99829.1 & 0.314229 & -0.01062 & -25.5143 & -0.47245 \\
\hline$(-100,-50,-150)$ & 107188.7 & 99826.9 & 0.295083 & 0.010396 & -24.9686 & -0.45662 \\
\hline$(-100,-100,-150)$ & 107186.6 & 99834.3 & 0.27534 & 0.007004 & -25.2318 & -0.48451 \\
\hline$(-100,-150,-150)$ & 107184.6 & 99829.3 & 0.284197 & 0.005699 & -25.6708 & -0.47177 \\
\hline
\end{tabular}




\begin{tabular}{lllllll}
$(-150,0,-150)$ & 107184.6 & 99826.9 & 0.27466 & -0.00069 & -24.9175 & -0.45777 \\
$(-150,-50,-150)$ & 107185.2 & 99829.4 & 0.296267 & 0.006989 & -25.1745 & -0.46775 \\
$(-150,-100,-150)$ & 107183.4 & 99831.5 & 0.287787 & -0.01316 & -24.9402 & -0.47206 \\
$(-150,-150,-150)$ & 107184.3 & 99828.7 & 0.282216 & -0.01127 & -25.3854 & -0.46939 \\
\hline
\end{tabular}

\section{Table 6}

Pressure values for probe C at $34.4 \mathrm{~m} / \mathrm{s}$

\begin{tabular}{|c|c|c|c|c|c|c|}
\hline Coordinate & $P_{11}$ & $P_{12}$ & $P_{13}$ & $P_{14}$ & $P_{15}$ & $\bar{P}$ \\
\hline$(0,0,0)$ & 99983.6 & 99649.8 & 99782.8 & 99667.3 & 99646.1 & 99686.5 \\
\hline$(50,0,0)$ & 99982.2 & 99646 & 99786.5 & 99669.4 & 99647.3 & 99687.32 \\
\hline$(50,50,0)$ & 99980.1 & 99647.3 & 99783.7 & 99667.9 & 99644.4 & 99685.83 \\
\hline$(50,100,0)$ & 99979.6 & 99644.4 & 99789.2 & 99667.3 & 99646.6 & 99686.86 \\
\hline$(50,150,0)$ & 99979.3 & 99646.6 & 99787.6 & 99669.3 & 99647.3 & 99687.69 \\
\hline$(100,0,0)$ & 99981.2 & 99647 & 99781.9 & 99669.0 & 99648.2 & 99686.61 \\
\hline$(100,50,0)$ & 99980.5 & 99648.2 & 99784.5 & 99666.9 & 99646.3 & 99686.48 \\
\hline$(100,100,0)$ & 99982.3 & 99646 & 99786.3 & 99670.6 & 99648.5 & 99687.86 \\
\hline$(100,150,0)$ & 99984.7 & 99647.3 & 99789.8 & 99669.6 & 99644.2 & 99687.73 \\
\hline$(150,0,0)$ & 99982.3 & 99644.4 & 99787.75 & 99668.0 & 99648 & 99687.08 \\
\hline$(150,50,0)$ & 99983.7 & 99646.6 & 99782.9 & 99668.7 & 99649.8 & 99687.01 \\
\hline$(150,100,0)$ & 99978.6 & 99647 & 99784.6 & 99668.8 & 99646.1 & 99686.71 \\
\hline$(150,150,0)$ & 99982.6 & 99648.2 & 99782.7 & 99668.8 & 99647.3 & 99686.76 \\
\hline$(-50,0,0)$ & 99978.1 & 99646.3 & 99788.8 & 99669.5 & 99644 & 99687.24 \\
\hline$(-50,-50,0)$ & 99983.7 & 99647.3 & 99782.7 & 99667.6 & 99646.6 & 99686.04 \\
\hline$(-50,-100,0)$ & 99981.5 & 99648.2 & 99781.5 & 99668.9 & 99647.3 & 99686.46 \\
\hline$(-50,-150,0)$ & 99980.7 & 99649.8 & 99785.3 & 99667.0 & 99648.2 & 99687.58 \\
\hline$(-100,0,0)$ & 99981.3 & 99648.5 & 99785.7 & 99669.8 & 99646.3 & 99687.58 \\
\hline$(-100,-50,0)$ & 99982.5 & 99644.2 & 99787.2 & 99667.0 & 99646.1 & 99686.13 \\
\hline$(-100,-100,0)$ & 99980.9 & 99648.2 & 99785.7 & 99669.0 & 99647.3 & 99687.54 \\
\hline$(-100,-150,0)$ & 99981.3 & 99649.8 & 99782.8 & 99667.9 & 99648.2 & 99687.17 \\
\hline$(-150,0,0)$ & 99980.7 & 99647.1 & 99786.5 & 99668.9 & 99646.3 & 99687.19 \\
\hline$(-150,-50,0)$ & 99980 & 99646.6 & 99783.7 & 99668.3 & 99648.5 & 99686.77 \\
\hline$(-150,-100,0)$ & 99982.7 & 99644.7 & 99789.2 & 99668.2 & 99644.2 & 99686.58 \\
\hline$(-150,-150,0)$ & 99983.7 & 99647.6 & 99787.6 & 99667.3 & 99646.1 & 99687.16 \\
\hline$(50,0,50)$ & 99980.3 & 99642.3 & 99781.9 & 99668.4 & 99647.3 & 99684.98 \\
\hline$(50,50,50)$ & 99977.4 & 99646.1 & 99784.5 & 99667.0 & 99648 & 99686.3 \\
\hline$(50,100,50)$ & 99984.8 & 99647.3 & 99786.3 & 99667.6 & 99648.5 & 99687.43 \\
\hline
\end{tabular}




\begin{tabular}{|c|c|c|c|c|c|c|}
\hline$(50,150,50)$ & 99982.9 & 99644.4 & 99789.8 & 99670.0 & 99644.2 & 99687.09 \\
\hline$(100,0,50)$ & 99979.7 & 99646.1 & 99787.75 & 99668.4 & 99648.2 & 99687.62 \\
\hline$(100,50,50)$ & 99981.3 & 99647.3 & 99782.9 & 99669.5 & 99649.8 & 99687.38 \\
\hline$(100,100,50)$ & 99983.7 & 99648.2 & 99784.6 & 99668.8 & 99647.1 & 99687.17 \\
\hline$(100,150,50)$ & 99984.7 & 99646.3 & 99782.7 & 99668.4 & 99647 & 99686.01 \\
\hline$(150,0,50)$ & 99982 & 99648.5 & 99785.1 & 99668.8 & 99644.7 & 99686.77 \\
\hline$(150,50,50)$ & 99983.7 & 99644.2 & 99789.2 & 99667.5 & 99647.6 & 99687.13 \\
\hline$(150,100,50)$ & 99978.6 & 99644.4 & 99781.5 & 99668.4 & 99642.3 & 99684.14 \\
\hline$(150,150,50)$ & 99985.2 & 99646.6 & 99785.3 & 99668.8 & 99646.1 & 99686.7 \\
\hline$(-50,0,-50)$ & 99982.6 & 99647 & 99785.7 & 99668.0 & 99647.3 & 99687.07 \\
\hline$(-50,-50,-50)$ & 99978.1 & 99648.2 & 99787.2 & 99669.3 & 99644.4 & 99687.26 \\
\hline$(-50,-100,-50)$ & 99983.7 & 99646.3 & 99785.7 & 99666.5 & 99646.6 & 99686.28 \\
\hline$(-50,-150,-50)$ & 99981.5 & 99647.3 & 99782.8 & 99667.6 & 99647.3 & 99686.24 \\
\hline$(-100,0,-50)$ & 99977.6 & 99648.2 & 99786.5 & 99669.4 & 99646 & 99687.54 \\
\hline$(-100,-50,-50)$ & 99983.6 & 99649.8 & 99783.7 & 99669.4 & 99647.7 & 99687.65 \\
\hline$(-100,-100,-50)$ & 99981.7 & 99648.5 & 99789.2 & 99670.9 & 99643.6 & 99688.05 \\
\hline$(-100,-150,-50)$ & 99983.4 & 99644.2 & 99784.5 & 99668.6 & 99646.3 & 99685.9 \\
\hline$(-150,0,-50)$ & 99982 & 99648.2 & 99786.3 & 99667.9 & 99645.4 & 99686.94 \\
\hline$(-150,-50,-50)$ & 99983.7 & 99649.8 & 99789.8 & 99667.3 & 99643 & 99687.56 \\
\hline$(-150,-100,-50)$ & 99981.4 & 99647.1 & 99787.5 & 99669.6 & 99647.6 & 99687.96 \\
\hline$(-150,-150,-50)$ & 99983.1 & 99646.6 & 99782.7 & 99666.4 & 99647.8 & 99685.86 \\
\hline$(50,0,100)$ & 99978.7 & 99644.7 & 99788.8 & 99668.8 & 99646.3 & 99687.15 \\
\hline$(-50,50,100)$ & 99982.9 & 99647.6 & 99782.7 & 99667.5 & 99647.1 & 99686.23 \\
\hline$(-50,100,100)$ & 99978.2 & 99642.3 & 99781.5 & 99669.3 & 99646.6 & 99684.91 \\
\hline$(50,150,100)$ & 99983.4 & 99646.1 & 99785.3 & 99667.0 & 99644.7 & 99685.78 \\
\hline$(100,0,100)$ & 99981.6 & 99647.3 & 99785.7 & 99669.0 & 99647.6 & 99687.41 \\
\hline$(100,50,100)$ & 99983.2 & 99644.4 & 99787.2 & 99668.2 & 99642.3 & 99685.52 \\
\hline$(100,100,100)$ & 99981 & 99646.1 & 99785.7 & 99668.1 & 99646.1 & 99686.5 \\
\hline$(100,150,100)$ & 99983.7 & 99644.7 & 99782.8 & 99668.4 & 99647.3 & 99685.79 \\
\hline$(150,0,100)$ & 99979.6 & 99647.6 & 99786.5 & 99668.9 & 99644.4 & 99686.84 \\
\hline$(150,50,100)$ & 99981.1 & 99642.3 & 99783.7 & 99669.0 & 99646.6 & 99685.39 \\
\hline$(150,100,100)$ & 99980.6 & 99646.1 & 99789.2 & 99668.3 & 99647.3 & 99687.72 \\
\hline$(150,150,100)$ & 99978.4 & 99647.3 & 99787.6 & 99669.5 & 99648.2 & 99688.14 \\
\hline$(-50,0,-100)$ & 99981.3 & 99644.4 & 99782.7 & 99668.8 & 99646.3 & 99685.54 \\
\hline$(-50,-50,-100)$ & 99980.7 & 99646.1 & 99781.5 & 99668.2 & 99648.5 & 99686.06 \\
\hline$(-50,-100,-100)$ & 99982.7 & 99647.3 & 99785.3 & 99669.8 & 99644.2 & 99686.64 \\
\hline$(-50,-150,-100)$ & 99981.1 & 99648.2 & 99785.7 & 99669.2 & 99648 & 99687.83 \\
\hline$(-100,0,-100)$ & 99983.7 & 99646.3 & 99787.2 & 99668.8 & 99649.8 & 99688.03 \\
\hline$(-100,-50,-100)$ & 99980 & 99648.5 & 99785.1 & 99669.9 & 99646.1 & 99687.39 \\
\hline$(-100,-100,-100)$ & 99984.8 & 99644.2 & 99789.2 & 99665.6 & 99647.3 & 99686.56 \\
\hline$(-100,-150,-100)$ & 99981.5 & 99644.4 & 99784.6 & 99668.1 & 99644 & 99685.38 \\
\hline
\end{tabular}




\begin{tabular}{|c|c|c|c|c|c|c|}
\hline$(-150,0,-100)$ & 99983.4 & 99646.6 & 99782.7 & 99668.7 & 99646.6 & 99686.14 \\
\hline$(-150,-50,-100)$ & 99982 & 99647 & 99788.8 & 99670.4 & 99647.3 & 99688.44 \\
\hline$(-150,-100,-100)$ & 99983.7 & 99648.2 & 99782.7 & 99668.0 & 99648.2 & 99686.78 \\
\hline$(-150,-150,-100)$ & 99981.4 & 99646.3 & 99781.5 & 99668.7 & 99646.3 & 99685.69 \\
\hline$(50,0,150)$ & 99983.1 & 99648.5 & 99785.3 & 99667.9 & 99646.1 & 99686.94 \\
\hline$(-50,50,150)$ & 99978.7 & 99646.1 & 99787.4 & 99669.4 & 99647.3 & 99687.56 \\
\hline$(-50,100,150)$ & 99982.9 & 99647.7 & 99786.3 & 99669.5 & 99648.2 & 99687.92 \\
\hline$(50,150,150)$ & 99983.4 & 99648.5 & 99785.1 & 99667.4 & 99646.3 & 99686.83 \\
\hline$(100,0,150)$ & 99982 & 99644.2 & 99789.2 & 99670.4 & 99648.5 & 99688.07 \\
\hline$(100,50,150)$ & 99983.7 & 99648.2 & 99784.6 & 99669.9 & 99644.2 & 99686.73 \\
\hline$(100,100,150)$ & 99981.4 & 99649.8 & 99782.7 & 99669.1 & 99648.2 & 99687.45 \\
\hline$(100,150,150)$ & 99983.1 & 99647.1 & 99785.1 & 99668.0 & 99649.8 & 99687.49 \\
\hline$(150,0,150)$ & 99978.7 & 99646.6 & 99789.2 & 99669.0 & 99648.2 & 99688.24 \\
\hline$(150,50,150)$ & 99982.9 & 99644.7 & 99784.6 & 99668.9 & 99646.3 & 99686.11 \\
\hline$(150,100,150)$ & 99983.4 & 99647.6 & 99782.7 & 99666.7 & 99648.5 & 99686.37 \\
\hline$(150,150,150)$ & 99982 & 99642.3 & 99788.8 & 99668.1 & 99644.2 & 99685.86 \\
\hline$(-50,0,-150)$ & 99983.7 & 99646.1 & 99782.7 & 99670.4 & 99648.2 & 99686.84 \\
\hline$(-50,-50,-150)$ & 99981.4 & 99647.3 & 99781.5 & 99668.9 & 99649.8 & 99686.86 \\
\hline$(-50,-100,-150)$ & 99983.1 & 99648.2 & 99785.3 & 99669.9 & 99647.1 & 99687.62 \\
\hline$(-50,-150,-150)$ & 99978.7 & 99649.8 & 99785.7 & 99667.4 & 99646.6 & 99687.38 \\
\hline$(-100,0,-150)$ & 99982.9 & 99646 & 99787.2 & 99668.1 & 99644.7 & 99686.51 \\
\hline$(-100,-50,-150)$ & 99978.2 & 99647.3 & 99785.7 & 99668.2 & 99647.6 & 99687.19 \\
\hline$(-100,-100,-150)$ & 99983.4 & 99644.4 & 99782.8 & 99669.2 & 99642.3 & 99684.68 \\
\hline$(-100,-150,-150)$ & 99981.6 & 99646.6 & 99786.5 & 99670.6 & 99646.1 & 99687.45 \\
\hline$(-150,0,-150)$ & 99983.2 & 99647 & 99783.7 & 99668.6 & 99647.3 & 99686.72 \\
\hline$(-150,-50,-150)$ & 99981 & 99648.2 & 99789.2 & 99667.7 & 99644.4 & 99687.36 \\
\hline$(-150,-100,-150)$ & 99983.7 & 99646 & 99787.6 & 99667.6 & 99647 & 99686.97 \\
\hline$(-150,-150,-150)$ & 99979.6 & 99647.3 & 99781.9 & 99668.7 & 99647.3 & 99686.31 \\
\hline
\end{tabular}

\section{Table 7}

Pressure Coefficient values for probe C at $34.4 \mathrm{~m} / \mathrm{s}$

\begin{tabular}{lllllll}
\hline Coordinate & $P_{\text {total (pitot) }}$ & $P_{\text {static }}$ pitot) & $C_{P_{\text {yaw }}}$ & $C_{P_{\text {pitch }}}$ & $C_{P_{\text {totat }}}$ & $C_{P_{\text {static }}}$ \\
\hline$(0,0,0)$ & 107185.2 & 99829.3 & -0.44766 & -0.07136 & -24.2397 & -0.48065 \\
$(50,0,0)$ & 107183.4 & 99825.7 & -0.47612 & -0.07486 & -24.4207 & -0.46928 \\
$(50,50,0)$ & 107187.6 & 99827.3 & -0.46352 & -0.07994 & -24.4929 & -0.48075 \\
$(50,100,0)$ & 107184.6 & 99822.8 & -0.49464 & -0.07054 & -24.6125 & -0.46437 \\
$(50,150,0)$ & 107183.6 & 99823.7 & -0.48352 & -0.07527 & -24.705 & -0.46642 \\
$(100,0,0)$ & 107184.7 & 99829.3 & -0.4569 & -0.07069 & -24.4523 & -0.48437
\end{tabular}




\begin{tabular}{|c|c|c|c|c|c|c|}
\hline$(100,50,0)$ & 107184.6 & 99830.2 & -0.46358 & -0.07015 & -24.5022 & -0.48881 \\
\hline$(100,100,0)$ & 107185.0 & 99831.5 & -0.47616 & -0.07489 & -24.4626 & -0.48784 \\
\hline$(100,150,0)$ & 107183.4 & 99828.7 & -0.47985 & -0.08562 & -24.2405 & -0.47469 \\
\hline$(150,0,0)$ & 107184.3 & 99833.9 & -0.48556 & -0.0669 & -24.395 & -0.49733 \\
\hline$(150,50,0)$ & 107188.7 & 99831.6 & -0.4594 & -0.06379 & -24.2843 & -0.48735 \\
\hline$(150,100,0)$ & 107186.6 & 99260.3 & -0.47038 & -0.07785 & -24.6939 & 1.460827 \\
\hline$(150,150,0)$ & 107184.6 & 99832.8 & -0.45463 & -0.07276 & -24.3439 & -0.49365 \\
\hline$(-50,0,0)$ & 107182.0 & 99829.1 & -0.48992 & -0.08612 & -24.7674 & -0.48773 \\
\hline$(-50,-50,0)$ & 107187.1 & 99826.9 & -0.45488 & -0.07038 & -24.1999 & -0.47323 \\
\hline$(-50,-100,0)$ & 107184.6 & 99834.3 & -0.45181 & -0.07304 & -24.4142 & -0.50108 \\
\hline$(-50,-150,0)$ & 107183.6 & 99829.3 & -0.46227 & -0.06422 & -24.5733 & -0.48349 \\
\hline$(-100,0,0)$ & 107184.7 & 99826.9 & -0.46711 & -0.08009 & -24.5248 & -0.47433 \\
\hline$(-100,-50,0)$ & 107184.6 & 99829.4 & -0.4825 & -0.07052 & -24.3006 & -0.48342 \\
\hline$(-100,-100,0)$ & 107183.4 & 99831.5 & -0.4687 & -0.0738 & -24.5514 & -0.49073 \\
\hline$(-100,-150,0)$ & 107184.3 & 99828.7 & -0.45218 & -0.06689 & -24.4891 & -0.48118 \\
\hline$(-150,0,0)$ & 107188.7 & 99833.9 & -0.47495 & -0.07691 & -24.5583 & -0.49984 \\
\hline$(-150,-50,0)$ & 107186.6 & 99831.6 & -0.46819 & -0.06753 & -24.6114 & -0.49459 \\
\hline$(-150,-100,0)$ & 107184.6 & 99260.3 & -0.48799 & -0.08118 & -24.3212 & 1.439588 \\
\hline$(-150,-150,0)$ & 107184.6 & 99832.8 & -0.47211 & -0.07157 & -24.2828 & -0.49114 \\
\hline$(50,0,50)$ & 107185.2 & 99834.3 & -0.4727 & -0.07145 & -24.3966 & -0.50563 \\
\hline$(50,50,50)$ & 107183.4 & 99833.9 & -0.47544 & -0.06664 & -24.7542 & -0.50704 \\
\hline$(50,100,50)$ & 107184.3 & 99832.8 & -0.46742 & -0.06423 & -24.2102 & -0.48886 \\
\hline$(50,150,50)$ & 107188.7 & 99832.8 & -0.49154 & -0.08713 & -24.3599 & -0.49257 \\
\hline$(100,0,50)$ & 107186.6 & 99831.6 & -0.48497 & -0.06924 & -24.6743 & -0.49295 \\
\hline$(100,50,50)$ & 107184.6 & 99831.5 & -0.46135 & -0.06711 & -24.5078 & -0.49034 \\
\hline$(100,100,50)$ & 107185.2 & 99829.4 & -0.45999 & -0.0731 & -24.2859 & -0.47965 \\
\hline$(100,150,50)$ & 107185.2 & 99829.3 & -0.45666 & -0.07307 & -24.1067 & -0.47973 \\
\hline$(150,0,50)$ & 107185.2 & 99829.1 & -0.46222 & -0.08146 & -24.3728 & -0.48161 \\
\hline$(150,50,50)$ & 107185.2 & 99828.7 & -0.48893 & -0.06719 & -24.2828 & -0.47736 \\
\hline$(150,100,50)$ & 107184.6 & 99826.9 & -0.46559 & -0.08847 & -24.4717 & -0.48482 \\
\hline$(150,150,50)$ & 107183.4 & 99826.9 & -0.46466 & -0.07605 & -24.1144 & -0.46968 \\
\hline$(-50,0,-50)$ & 107184.3 & 99260.3 & -0.46831 & -0.06996 & -24.3687 & 1.444073 \\
\hline$(-50,-50,-50)$ & 107188.7 & 99834.3 & -0.47793 & -0.08544 & -24.7925 & -0.50557 \\
\hline$(-50,-100,-50)$ & 107186.6 & 99834.3 & -0.4687 & -0.06699 & -24.218 & -0.49768 \\
\hline$(-50,-150,-50)$ & 107184.6 & 99833.9 & -0.45891 & -0.06858 & -24.3956 & -0.50011 \\
\hline$(-100,0,-50)$ & 107184.6 & 99833.9 & -0.4768 & -0.08024 & -24.8469 & -0.50458 \\
\hline$(-100,-50,-50)$ & 107185.2 & 99832.8 & -0.45244 & -0.07332 & -24.3339 & -0.49045 \\
\hline$(-100,-100,-50)$ & 107183.4 & 99832.8 & -0.47915 & -0.09301 & -24.5249 & -0.49293 \\
\hline$(-100,-150,-50)$ & 107184.3 & 99832.8 & -0.4716 & -0.07496 & -24.2047 & -0.49378 \\
\hline$(-150,0,-50)$ & 107188.7 & 99831.6 & -0.46772 & -0.07603 & -24.4071 & -0.48995 \\
\hline$(-150,-50,-50)$ & 107185.2 & 99831.6 & -0.47275 & -0.08054 & -24.3182 & -0.48639 \\
\hline
\end{tabular}




\begin{tabular}{|c|c|c|c|c|c|c|}
\hline$(-150,-100,-50)$ & 107184.6 & 99831.5 & -0.47846 & -0.07506 & -24.5471 & -0.48917 \\
\hline$(-150,-150,-50)$ & 107183.4 & 99825.7 & -0.45788 & -0.06241 & -24.2239 & -0.47046 \\
\hline$(50,0,100)$ & 107184.3 & 99827.3 & -0.49425 & -0.07717 & -24.7148 & -0.48071 \\
\hline$(-50,50,100)$ & 107188.7 & 99822.8 & -0.45539 & -0.06885 & -24.289 & -0.46034 \\
\hline$(-50,100,100)$ & 107186.6 & 99823.7 & -0.47462 & -0.07723 & -24.5779 & -0.47321 \\
\hline$(50,150,100)$ & 107184.6 & 99829.3 & -0.46771 & -0.07501 & -24.1961 & -0.48222 \\
\hline$(100,0,100)$ & 107184.6 & 99830.2 & -0.47044 & -0.07283 & -24.4839 & -0.48537 \\
\hline$(100,50,100)$ & 107185.2 & 99831.5 & -0.47971 & -0.08692 & -24.1938 & -0.49039 \\
\hline$(100,100,100)$ & 107183.4 & 99828.7 & -0.47386 & -0.07468 & -24.4476 & -0.48269 \\
\hline$(100,150,100)$ & 107184.3 & 99833.9 & -0.46356 & -0.07066 & -24.1702 & -0.49717 \\
\hline$(150,0,100)$ & 107188.7 & 99831.6 & -0.47446 & -0.0836 & -24.6249 & -0.49446 \\
\hline$(150,50,100)$ & 107184.3 & 99260.3 & -0.47818 & -0.07567 & -24.3593 & 1.437554 \\
\hline$(150,100,100)$ & 107188.7 & 99832.8 & -0.48859 & -0.07157 & -24.6107 & -0.49536 \\
\hline$(150,150,100)$ & 107186.6 & 99829.1 & -0.48337 & -0.0733 & -24.8339 & -0.48563 \\
\hline$(-50,0,-100)$ & 107184.6 & 99826.9 & -0.4676 & -0.07591 & -24.355 & -0.47796 \\
\hline$(-50,-50,-100)$ & 107184.6 & 99834.3 & -0.45955 & -0.06669 & -24.45 & -0.50312 \\
\hline$(-50,-100,-100)$ & 107185.2 & 99829.3 & -0.46613 & -0.08639 & -24.3282 & -0.48186 \\
\hline$(-50,-150,-100)$ & 107183.4 & 99826.9 & -0.46884 & -0.07161 & -24.558 & -0.47421 \\
\hline$(-100,0,-100)$ & 107184.3 & 99829.4 & -0.47655 & -0.06435 & -24.3536 & -0.47813 \\
\hline$(-100,-50,-100)$ & 107188.7 & 99831.5 & -0.46635 & -0.08108 & -24.6094 & -0.492 \\
\hline$(-100,-100,-100)$ & 107185.2 & 99828.7 & -0.48619 & -0.06119 & -24.1433 & -0.47659 \\
\hline$(-100,-150,-100)$ & 107188.7 & 99833.9 & -0.47345 & -0.08003 & -24.3384 & -0.50156 \\
\hline$(-150,0,-100)$ & 107185.2 & 99826.9 & -0.45784 & -0.07418 & -24.2272 & -0.47353 \\
\hline$(-150,-50,-100)$ & 107184.6 & 99832.8 & -0.48169 & -0.07855 & -24.5183 & -0.49142 \\
\hline$(-150,-100,-100)$ & 107183.4 & 99832.8 & -0.45298 & -0.06673 & -24.2477 & -0.49179 \\
\hline$(-150,-150,-100)$ & 107184.3 & 99831.6 & -0.45721 & -0.07567 & -24.3583 & -0.49342 \\
\hline$(50,0,150)$ & 107188.7 & 99831.5 & -0.46191 & -0.07344 & -24.3299 & -0.48812 \\
\hline$(-50,50,150)$ & 107186.6 & 99829.4 & -0.48533 & -0.07599 & -24.7572 & -0.48719 \\
\hline$(-50,100,150)$ & 107184.6 & 99829.4 & -0.46986 & -0.07212 & -24.4141 & -0.47963 \\
\hline$(50,150,150)$ & 107184.6 & 99829.3 & -0.4606 & -0.07123 & -24.2817 & -0.48039 \\
\hline$(100,0,150)$ & 107185.2 & 99825.7 & -0.49298 & -0.07437 & -24.4892 & -0.46792 \\
\hline$(100,50,150)$ & 107183.4 & 99827.3 & -0.45931 & -0.08663 & -24.2438 & -0.47335 \\
\hline$(100,100,150)$ & 107184.3 & 99822.8 & -0.45212 & -0.0711 & -24.5038 & -0.46045 \\
\hline$(100,150,150)$ & 107188.7 & 99823.7 & -0.46684 & -0.06148 & -24.3757 & -0.46077 \\
\hline$(150,0,150)$ & 107185.2 & 99829.3 & -0.49095 & -0.07153 & -24.8111 & -0.48564 \\
\hline$(150,50,150)$ & 107184.3 & 99830.2 & -0.47138 & -0.07598 & -24.2645 & -0.48549 \\
\hline$(150,100,150)$ & 107185.2 & 99831.5 & -0.45483 & -0.06119 & -24.246 & -0.48861 \\
\hline$(150,150,150)$ & 107185.2 & 99828.7 & -0.49436 & -0.08073 & -24.3063 & -0.48202 \\
\hline$(-50,0,-150)$ & 107188.7 & 99833.9 & -0.46016 & -0.0747 & -24.271 & -0.49538 \\
\hline$(-50,-50,-150)$ & 107185.2 & 99831.6 & -0.45563 & -0.06468 & -24.4581 & -0.49141 \\
\hline$(-50,-100,-150)$ & 107184.6 & 99260.3 & -0.46398 & -0.07703 & -24.3718 & 1.446153 \\
\hline
\end{tabular}




\begin{tabular}{lllllll}
$(-50,-150,-150)$ & 107183.4 & 99832.8 & -0.46649 & -0.0714 & -24.7307 & -0.49918 \\
$(-100,0,-150)$ & 107184.3 & 99829.1 & -0.47607 & -0.07878 & -24.2972 & -0.48108 \\
$(-100,-50,-150)$ & 107188.7 & 99826.9 & -0.47559 & -0.0707 & -24.7778 & -0.48008 \\
$(-100,-100,-150)$ & 107186.6 & 99834.3 & -0.46331 & -0.09013 & -24.1137 & -0.50087 \\
$(-100,-150,-150)$ & 107184.6 & 99829.3 & -0.4756 & -0.08325 & -24.4872 & -0.48224 \\
$(-150,0,-150)$ & 107184.6 & 99826.9 & -0.46006 & -0.07176 & -24.2896 & -0.47282 \\
$(-150,-50,-150)$ & 107185.2 & 99829.4 & -0.48002 & -0.07915 & -24.5257 & -0.48355 \\
$(-150,-100,-150)$ & 107183.4 & 99831.5 & -0.47686 & -0.07069 & -24.2632 & -0.48708 \\
$(-150,-150,-150)$ & 107184.3 & 99828.7 & -0.45893 & -0.07305 & -24.5648 & -0.4855 \\
\hline
\end{tabular}

\section{Table 8}

Pressure values for probe D at $34.4 \mathrm{~m} / \mathrm{s}$

\begin{tabular}{llrcrrr}
\hline Coordinate & $P_{16}$ & $P_{17}$ & $P_{18}$ & $P_{19}$ & $P_{20}$ & $\bar{P}$ \\
\hline$(0,0,0)$ & 99980 & 99785.1 & 99649.8 & 99694.6 & 99648.5 & 99694.5 \\
$(50,0,0)$ & 99984.8 & 99789.2 & 99647.1 & 99694.3 & 99644.2 & 99693.7 \\
$(50,50,0)$ & 99981.5 & 99781.5 & 99646.6 & 99696.8 & 99648.2 & 99693.27 \\
$(50,100,0)$ & 99983.4 & 99785.3 & 99644.7 & 99696.7 & 99649.8 & 99694.13 \\
$(50,150,0)$ & 99982 & 99785.7 & 99647.6 & 99696.1 & 99647.1 & 99694.13 \\
$(100,0,0)$ & 99983.7 & 99787.2 & 99642.3 & 99694.9 & 99646.6 & 99692.76 \\
$(100,50,0)$ & 99981.4 & 99785.7 & 99646.1 & 99694.3 & 99644.7 & 99692.69 \\
$(100,100,0)$ & 99983.1 & 99782.8 & 99647.3 & 99695.9 & 99647.6 & 99693.4 \\
$(100,150,0)$ & 99978.7 & 99786.3 & 99648.2 & 99696.1 & 99642.3 & 99693.22 \\
$(150,0,0)$ & 99982.9 & 99789.8 & 99649.8 & 99697.2 & 99648.5 & 99696.32 \\
$(150,50,0)$ & 99978.2 & 99787.5 & 99646 & 99695.8 & 99646.1 & 99693.87 \\
$(150,100,0)$ & 99983.4 & 99782.7 & 99647.6 & 99695.0 & 99647.7 & 99693.25 \\
$(150,150,0)$ & 99981.6 & 99788.8 & 99642.3 & 99695.6 & 99648.5 & 99693.8 \\
$(-50,0,0)$ & 99983.2 & 99782.7 & 99646.1 & 99696.1 & 99644.2 & 99692.28 \\
$(-50,-50,0)$ & 99982.7 & 99781.5 & 99647.3 & 99696.4 & 99648.2 & 99693.36 \\
$(-50,-100,0)$ & 99981.1 & 99785.3 & 99648.2 & 99696.0 & 99649.8 & 99694.82 \\
$(-50,-150,0)$ & 99983.7 & 99785.7 & 99649.8 & 99694.7 & 99647.1 & 99694.31 \\
$(-100,0,0)$ & 99980 & 99787.2 & 99646 & 99695.6 & 99646.6 & 99693.87 \\
$(-100,-50,0)$ & 99984.8 & 99785.7 & 99647.3 & 99695.7 & 99644.7 & 99693.34 \\
$(-100,-100,0)$ & 99981.5 & 99782.8 & 99644.4 & 99696.1 & 99647.6 & 99692.73 \\
$(-100,-150,0)$ & 99983.4 & 99786.5 & 99646.6 & 99696.6 & 99642.3 & 99693
\end{tabular}




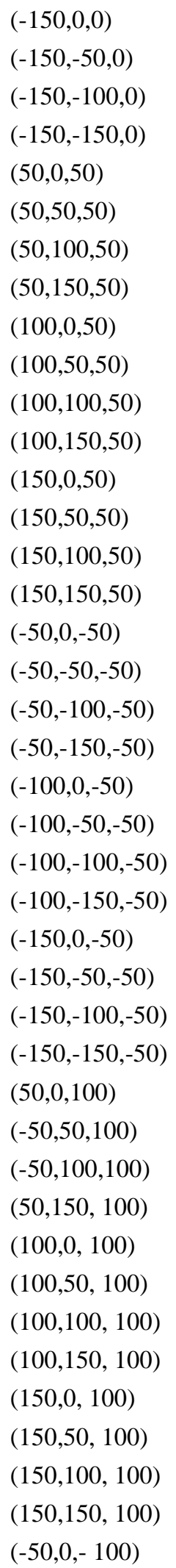

\begin{tabular}{|c|c|c|c|c|c|}
\hline 99982 & 99783.7 & 99647 & 99696.9 & 99646.1 & 99693.51 \\
\hline 99983.7 & 99789.2 & 99648.2 & 99695.2 & 99647.3 & 99694.99 \\
\hline 99981.4 & 99787.6 & 99646.3 & 99695.4 & 99648.2 & 99694.38 \\
\hline 99983.1 & 99782.7 & 99645.4 & 99697.2 & 99649.8 & 99693.77 \\
\hline 99978.7 & 99781.5 & 99643 & 99696.2 & 99646 & 99691.8 \\
\hline 99982.9 & 99785.3 & 99647.6 & 99694.5 & 99647.3 & 99693.68 \\
\hline 99978.2 & 99785.7 & 99647.8 & 99696.4 & 99644.4 & 99693.57 \\
\hline 99983.4 & 99787.2 & 99646.3 & 99694.6 & 99644.4 & 99693.11 \\
\hline 99981.6 & 99785.1 & 99647.1 & 99693.7 & 99646.1 & 99693 \\
\hline 99983.2 & 99789.2 & 99646.6 & 99695.2 & 99647.3 & 99694.57 \\
\hline 99981 & 99784.6 & 99644.7 & 99696.2 & 99648.2 & 99693.42 \\
\hline 99983.7 & 99782.7 & 99647.6 & 99695.3 & 99646.3 & 99692.98 \\
\hline 99979.6 & 99788.8 & 99642.3 & 99695.0 & 99648.5 & 99693.66 \\
\hline 99981.1 & 99782.7 & 99646.1 & 99695.5 & 99644.2 & 99692.12 \\
\hline 99980.6 & 99781.5 & 99647.3 & 99694.8 & 99644.4 & 99692.01 \\
\hline 99979 & 99785.3 & 99644.4 & 99694.6 & 99646.6 & 99692.74 \\
\hline 99982.7 & 99787.4 & 99646.6 & 99697.5 & 99647 & 99694.69 \\
\hline 99983.9 & 99786.3 & 99647.3 & 99694.7 & 99648.2 & 99694.11 \\
\hline 99980.3 & 99785.1 & 99648.2 & 99695.1 & 99646.3 & 99693.67 \\
\hline 99977.4 & 99789.2 & 99646.3 & 99696.0 & 99647.3 & 99694.69 \\
\hline 99982.2 & 99784.6 & 99648.5 & 99696.7 & 99648.2 & 99694.5 \\
\hline 99983.6 & 99782.7 & 99644.2 & 99696.1 & 99649.8 & 99693.19 \\
\hline 99982.2 & 99785.1 & 99648 & 99697.1 & 99648.5 & 99694.73 \\
\hline 99980.1 & 99789.2 & 99649.8 & 99694.5 & 99644.2 & 99694.44 \\
\hline 99979.6 & 99784.6 & 99646.1 & 99696.2 & 99648.2 & 99693.77 \\
\hline 99979.3 & 99782.7 & 99647.3 & 99696.4 & 99649.8 & 99694.05 \\
\hline 99981.2 & 99785.7 & 99644 & 99694.9 & 99647.1 & 99693.03 \\
\hline 99980.5 & 99787.2 & 99646.6 & 99695.9 & 99646.6 & 99694.08 \\
\hline 99982.3 & 99785.7 & 99647.3 & 99694.8 & 99644.7 & 99693.12 \\
\hline 99984.7 & 99782.8 & 99648.2 & 99696.1 & 99647.6 & 99693.68 \\
\hline 99982.3 & 99786.3 & 99646.3 & 99695.3 & 99642.3 & 99692.55 \\
\hline 99983.7 & 99789.8 & 99646.1 & 99696.3 & 99646.1 & 99694.57 \\
\hline 99978.6 & 99787.5 & 99647.3 & 99694.6 & 99649.8 & 99694.81 \\
\hline 99982.6 & 99782.7 & 99648.2 & 99695.7 & 99646 & 99693.17 \\
\hline 99978.1 & 99788.8 & 99646.3 & 99695.4 & 99647.3 & 99694.45 \\
\hline 99983.7 & 99782.7 & 99648.5 & 99695.6 & 99644.4 & 99692.81 \\
\hline 99981.5 & 99781.5 & 99644.2 & 99695.7 & 99646.6 & 99691.99 \\
\hline 99980.7 & 99785.3 & 99648.2 & 99696.9 & 99647 & 99694.43 \\
\hline 99981.3 & 99785.7 & 99649.8 & 99695.2 & 99648.2 & 99694.72 \\
\hline 99982.5 & 99785.3 & 99648.2 & 99696.3 & 99646 & 99693.99 \\
\hline 99980.9 & 99787.4 & 99646.3 & 99695.0 & 99647.3 & 99694 \\
\hline
\end{tabular}




\begin{tabular}{|c|c|c|c|c|c|c|}
\hline$(-50,-50,-100)$ & 99981.3 & 99786.3 & 99647.1 & 99695.7 & 99644.4 & 99693.36 \\
\hline$(-50,-100,-100)$ & 99980.7 & 99785.1 & 99646.6 & 99695.4 & 99646.6 & 99693.42 \\
\hline$(-50,-150,-100)$ & 99980 & 99789.2 & 99644.7 & 99697.1 & 99647 & 99694.58 \\
\hline$(-100,0,-100)$ & 99982.7 & 99784.6 & 99647.6 & 99694.4 & 99648.2 & 99693.69 \\
\hline$(-100,-50,-100)$ & 99983.7 & 99782.7 & 99642.3 & 99695.9 & 99646.3 & 99691.8 \\
\hline$(-100,-100,-100)$ & 99980.3 & 99785.1 & 99646.1 & 99697.1 & 99647.3 & 99693.9 \\
\hline$(-100,-150,-100)$ & 99977.4 & 99789.2 & 99647.3 & 99696.4 & 99648.2 & 99695.28 \\
\hline$(-150,0,-100)$ & 99984.8 & 99784.6 & 99644.4 & 99695.2 & 99649.8 & 99693.5 \\
\hline$(-150,-50,-100)$ & 99982.9 & 99782.7 & 99646.6 & 99695.0 & 99648.5 & 99693.19 \\
\hline$(-150,-100,-100)$ & 99979.7 & 99785.7 & 99647.3 & 99694.9 & 99644.2 & 99693.03 \\
\hline$(-150,-150,-100)$ & 99981.3 & 99787.2 & 99648.2 & 99694.8 & 99648.2 & 99694.6 \\
\hline$(50,0,150)$ & 99983.7 & 99785.7 & 99646.3 & 99696.3 & 99649.8 & 99694.53 \\
\hline$(-50,50,150)$ & 99984.7 & 99782.8 & 99648.5 & 99695.6 & 99647.1 & 99693.5 \\
\hline$(-50,100,150)$ & 99982 & 99786.3 & 99644.2 & 99694.8 & 99646.6 & 99692.98 \\
\hline$(50,150,150)$ & 99983.7 & 99789.8 & 99648 & 99694.7 & 99644.7 & 99694.34 \\
\hline$(100,0,150)$ & 99978.6 & 99787.5 & 99649.8 & 99695.9 & 99647.6 & 99695.19 \\
\hline$(100,50,150)$ & 99985.2 & 99787.2 & 99646.1 & 99694.9 & 99642.3 & 99692.63 \\
\hline$(100,100,150)$ & 99982.6 & 99785.7 & 99647.3 & 99697.1 & 99646.1 & 99694.04 \\
\hline$(100,150,150)$ & 99978.1 & 99782.8 & 99644 & 99696.0 & 99647 & 99692.63 \\
\hline$(150,0,150)$ & 99983.7 & 99786.5 & 99646.6 & 99695.4 & 99648.2 & 99694.18 \\
\hline$(150,50,150)$ & 99981.5 & 99783.7 & 99647.3 & 99695.7 & 99646.3 & 99693.25 \\
\hline$(150,100,150)$ & 99977.6 & 99789.2 & 99648.2 & 99694.9 & 99647.3 & 99694.91 \\
\hline$(150,150,150)$ & 99983.6 & 99787.6 & 99646.3 & 99695.3 & 99648.2 & 99694.36 \\
\hline$(-50,0,-150)$ & 99981.7 & 99781.9 & 99646.1 & 99695.5 & 99649.8 & 99693.33 \\
\hline$(-50,-50,-150)$ & 99980 & 99787.2 & 99647.3 & 99695.9 & 99648.5 & 99694.73 \\
\hline$(-50,-100,-150)$ & 99984.8 & 99785.7 & 99648.2 & 99695.3 & 99644.2 & 99693.36 \\
\hline$(-50,-150,-150)$ & 99981.5 & 99782.8 & 99646.3 & 99695.5 & 99648.2 & 99693.21 \\
\hline$(-100,0,-150)$ & 99983.4 & 99786.3 & 99648.5 & 99694.9 & 99649.8 & 99694.88 \\
\hline$(-100,-50,-150)$ & 99982 & 99789.8 & 99644.2 & 99695.9 & 99647.1 & 99694.25 \\
\hline$(-100,-100,-150)$ & 99983.7 & 99787.5 & 99648.2 & 99695.4 & 99646.6 & 99694.44 \\
\hline$(-100,-150,-150)$ & 99981.4 & 99787.2 & 99649.8 & 99696.9 & 99644.7 & 99694.64 \\
\hline$(-150,0,-150)$ & 99983.1 & 99785.7 & 99648.2 & 99694.6 & 99647.6 & 99694.03 \\
\hline$(-150,-50,-150)$ & 99978.7 & 99782.8 & 99646.3 & 99696.1 & 99642.3 & 99691.86 \\
\hline$(-150,-100,-150)$ & 99982.9 & 99786.5 & 99648.5 & 99695.2 & 99646.1 & 99694.08 \\
\hline$(-150,-150,-150)$ & 99978.2 & 99787.6 & 99644.2 & 99695.5 & 99649.8 & 99694.27 \\
\hline
\end{tabular}

\section{Table 9}

Pressure Coefficient values for probe D at $34.4 \mathrm{~m} / \mathrm{s}$ 


\begin{tabular}{|c|c|c|c|c|c|c|}
\hline Coordinate & $P_{\text {total (pitot) }}$ & $P_{\text {static(pitot) }}$ & $C_{P_{\text {yaw }}}$ & $C_{P_{\text {pitch }}}$ & $C_{P_{\text {total }}}$ & $C_{P_{\text {static }}}$ \\
\hline$(0,0,0)$ & 107185.2 & 99829.3 & 0.473402 & -0.16125 & -25.2094 & -0.47166 \\
\hline$(50,0,0)$ & 107183.4 & 99825.7 & 0.488156 & -0.17217 & -24.7294 & -0.45344 \\
\hline$(50,50,0)$ & 107187.6 & 99827.3 & 0.46803 & -0.16855 & -25.0012 & -0.46501 \\
\hline$(50,100,0)$ & 107184.6 & 99822.8 & 0.486045 & -0.16215 & -24.8941 & -0.44482 \\
\hline$(50,150,0)$ & 107183.6 & 99823.7 & 0.479397 & -0.17016 & -24.9988 & -0.44979 \\
\hline$(100,0,0)$ & 107184.7 & 99829.3 & 0.498037 & -0.16612 & -24.7506 & -0.46931 \\
\hline$(100,50,0)$ & 107184.6 & 99830.2 & 0.483526 & -0.17162 & -24.9494 & -0.4763 \\
\hline$(100,100,0)$ & 107185.0 & 99831.5 & 0.467733 & -0.16679 & -24.8603 & -0.47669 \\
\hline$(100,150,0)$ & 107183.4 & 99828.7 & 0.48375 & -0.18841 & -25.2372 & -0.47457 \\
\hline$(150,0,0)$ & 107184.3 & 99833.9 & 0.488523 & -0.16989 & -25.1289 & -0.48007 \\
\hline$(150,50,0)$ & 107188.7 & 99831.6 & 0.49731 & -0.17473 & -25.3596 & -0.4844 \\
\hline$(150,100,0)$ & 107186.6 & 99260.3 & 0.465621 & -0.16302 & -24.8258 & 1.492159 \\
\hline$(150,150,0)$ & 107184.6 & 99832.8 & 0.509037 & -0.16368 & -25.0279 & -0.48297 \\
\hline$(-50,0,0)$ & 107182.0 & 99829.1 & 0.469542 & -0.17844 & -24.7448 & -0.4703 \\
\hline$(-50,-50,0)$ & 107187.1 & 99826.9 & 0.463808 & -0.16667 & -24.8991 & -0.46154 \\
\hline$(-50,-100,0)$ & 107184.6 & 99834.3 & 0.4789 & -0.16129 & -25.1623 & -0.48722 \\
\hline$(-50,-150,0)$ & 107183.6 & 99829.3 & 0.469613 & -0.16431 & -24.8798 & -0.46646 \\
\hline$(-100,0,0)$ & 107184.7 & 99826.9 & 0.492608 & -0.17094 & -25.152 & -0.46445 \\
\hline$(-100,-50,0)$ & 107184.6 & 99829.4 & 0.474847 & -0.17481 & -24.7023 & -0.46683 \\
\hline$(-100,-100,0)$ & 107183.4 & 99831.5 & 0.479274 & -0.16802 & -24.9398 & -0.48056 \\
\hline$(-100,-150,0)$ & 107184.3 & 99828.7 & 0.481742 & -0.18692 & -24.7961 & -0.4673 \\
\hline$(-150,0,0)$ & 107188.7 & 99833.9 & 0.472481 & -0.17612 & -24.9628 & -0.4863 \\
\hline$(-150,-50,0)$ & 107186.6 & 99831.6 & 0.488372 & -0.16606 & -24.9482 & -0.47318 \\
\hline$(-150,-100,0)$ & 107184.6 & 99260.3 & 0.492305 & -0.16456 & -25.0968 & 1.512393 \\
\hline$(-150,-150,0)$ & 107184.6 & 99832.8 & 0.47455 & -0.16381 & -24.8906 & -0.48052 \\
\hline$(50,0,50)$ & 107185.2 & 99834.3 & 0.481352 & -0.17463 & -25.1186 & -0.49669 \\
\hline$(50,50,50)$ & 107183.4 & 99833.9 & 0.476102 & -0.16322 & -24.8958 & -0.48483 \\
\hline$(50,100,50)$ & 107184.3 & 99832.8 & 0.484481 & -0.18256 & -25.317 & -0.48917 \\
\hline$(50,150,50)$ & 107188.7 & 99832.8 & 0.485381 & -0.17276 & -24.8213 & -0.4812 \\
\hline$(100,0,50)$ & 107186.6 & 99831.6 & 0.478165 & -0.16489 & -24.9651 & -0.48026 \\
\hline$(100,50,50)$ & 107184.6 & 99831.5 & 0.494056 & -0.16587 & -24.9502 & -0.47442 \\
\hline$(100,100,50)$ & 107185.2 & 99829.4 & 0.486299 & -0.16674 & -25.0419 & -0.47268 \\
\hline$(100,150,50)$ & 107185.2 & 99829.3 & 0.464703 & -0.16857 & -24.7711 & -0.46891 \\
\hline$(150,0,50)$ & 107185.2 & 99829.1 & 0.512347 & -0.16278 & -25.1999 & -0.47366 \\
\hline$(150,50,50)$ & 107185.2 & 99828.7 & 0.472692 & -0.17741 & -24.9293 & -0.47263 \\
\hline$(150,100,50)$ & 107184.6 & 99826.9 & 0.465016 & -0.17475 & -24.9626 & -0.46741 \\
\hline$(150,150,50)$ & 107183.4 & 99826.9 & 0.491516 & -0.1676 & -25.1303 & -0.46802 \\
\hline$(-50,0,-50)$ & 107184.3 & 99260.3 & 0.488878 & -0.17421 & -25.005 & 1.508279 \\
\hline$(-50,-50,-50)$ & 107188.7 & 99834.3 & 0.479664 & -0.16031 & -24.8625 & -0.48376 \\
\hline$(-50,-100,-50)$ & 107186.6 & 99834.3 & 0.477622 & -0.17021 & -25.1416 & -0.49063 \\
\hline
\end{tabular}




\begin{tabular}{|c|c|c|c|c|c|c|}
\hline$(-50,-150,-50)$ & 107184.6 & 99833.9 & 0.505472 & -0.17218 & -25.4936 & -0.49241 \\
\hline$(-100,0,-50)$ & 107184.6 & 99833.9 & 0.473065 & -0.1686 & -25.0345 & -0.48453 \\
\hline$(-100,-50,-50)$ & 107185.2 & 99832.8 & 0.476918 & -0.15935 & -24.7985 & -0.48073 \\
\hline$(-100,-100,-50)$ & 107183.4 & 99832.8 & 0.476231 & -0.16919 & -25.0505 & -0.48029 \\
\hline$(-100,-150,-50)$ & 107184.3 & 99832.8 & 0.487986 & -0.17623 & -25.2191 & -0.48436 \\
\hline$(-150,0,-50)$ & 107188.7 & 99831.6 & 0.484549 & -0.16782 & -25.2214 & -0.48221 \\
\hline$(-150,-50,-50)$ & 107185.2 & 99831.6 & 0.474674 & -0.16339 & -25.2619 & -0.48221 \\
\hline$(-150,-100,-50)$ & 107184.6 & 99831.5 & 0.49033 & -0.16589 & -24.9968 & -0.48052 \\
\hline$(-150,-150,-50)$ & 107183.4 & 99825.7 & 0.490892 & -0.17224 & -25.1481 & -0.45953 \\
\hline$(50,0,100)$ & 107184.3 & 99827.3 & 0.478598 & -0.17321 & -24.9051 & -0.464 \\
\hline$(-50,50,100)$ & 107188.7 & 99822.8 & 0.462503 & -0.16665 & -24.7539 & -0.44369 \\
\hline$(-50,100,100)$ & 107186.6 & 99823.7 & 0.483173 & -0.18289 & -24.8637 & -0.45263 \\
\hline$(50,150,100)$ & 107184.6 & 99829.3 & 0.497003 & -0.17351 & -24.9052 & -0.46599 \\
\hline$(100,0,100)$ & 107184.6 & 99830.2 & 0.494029 & -0.15802 & -25.3921 & -0.47708 \\
\hline$(100,50,100)$ & 107185.2 & 99831.5 & 0.46471 & -0.17133 & -24.8857 & -0.47794 \\
\hline$(100,100,100)$ & 107183.4 & 99828.7 & 0.502385 & -0.16962 & -25.4022 & -0.47329 \\
\hline$(100,150,100)$ & 107184.3 & 99833.9 & 0.461342 & -0.17614 & -24.7536 & -0.48503 \\
\hline$(150,0,100)$ & 107188.7 & 99831.6 & 0.474251 & -0.16947 & -24.8945 & -0.48223 \\
\hline$(150,50,100)$ & 107184.3 & 99260.3 & 0.478921 & -0.17335 & -25.1638 & 1.516516 \\
\hline$(150,100,100)$ & 107188.7 & 99832.8 & 0.474216 & -0.16396 & -25.1499 & -0.48182 \\
\hline$(150,150,100)$ & 107186.6 & 99829.1 & 0.475193 & -0.17415 & -24.9697 & -0.46831 \\
\hline$(-50,0,-100)$ & 107184.6 & 99826.9 & 0.491801 & -0.16619 & -25.1083 & -0.46324 \\
\hline$(-50,-50,-100)$ & 107184.6 & 99834.3 & 0.483441 & -0.17801 & -25.017 & -0.48947 \\
\hline$(-50,-100,-100)$ & 107185.2 & 99829.3 & 0.482103 & -0.16976 & -25.0782 & -0.47299 \\
\hline$(-50,-150,-100)$ & 107183.4 & 99826.9 & 0.506979 & -0.17477 & -25.2744 & -0.46425 \\
\hline$(-100,0,-100)$ & 107184.3 & 99829.4 & 0.474028 & -0.15968 & -24.918 & -0.46957 \\
\hline$(-100,-50,-100)$ & 107188.7 & 99831.5 & 0.480987 & -0.16992 & -24.6831 & -0.47859 \\
\hline$(-100,-100,-100)$ & 107185.2 & 99828.7 & 0.485335 & -0.17388 & -25.1569 & -0.47067 \\
\hline$(-100,-150,-100)$ & 107188.7 & 99833.9 & 0.50298 & -0.17094 & -25.5612 & -0.49135 \\
\hline$(-150,0,-100)$ & 107185.2 & 99826.9 & 0.481299 & -0.15592 & -24.7186 & -0.45794 \\
\hline$(-150,-50,-100)$ & 107184.6 & 99832.8 & 0.469781 & -0.16038 & -24.8584 & -0.48189 \\
\hline$(-150,-100,-100)$ & 107183.4 & 99832.8 & 0.482787 & -0.17695 & -25.1289 & -0.48756 \\
\hline$(-150,-150,-100)$ & 107184.3 & 99831.6 & 0.484827 & -0.16254 & -25.1238 & -0.47785 \\
\hline$(50,0,150)$ & 107188.7 & 99831.5 & 0.482071 & -0.16089 & -24.9162 & -0.47366 \\
\hline$(-50,50,150)$ & 107186.6 & 99829.4 & 0.461195 & -0.16655 & -24.7318 & -0.46669 \\
\hline$(-50,100,150)$ & 107184.6 & 99829.4 & 0.491151 & -0.16666 & -24.8939 & -0.47152 \\
\hline$(50,150,150)$ & 107184.6 & 99829.3 & 0.489357 & -0.17267 & -24.8857 & -0.46641 \\
\hline$(100,0,150)$ & 107185.2 & 99825.7 & 0.485875 & -0.17034 & -25.4286 & -0.46049 \\
\hline$(100,50,150)$ & 107183.4 & 99827.3 & 0.482277 & -0.17985 & -24.6032 & -0.4603 \\
\hline$(100,100,150)$ & 107184.3 & 99822.8 & 0.47962 & -0.17658 & -24.9572 & -0.44622 \\
\hline$(100,150,150)$ & 107188.7 & 99823.7 & 0.484809 & -0.17062 & -25.2584 & -0.45914 \\
\hline
\end{tabular}




\begin{tabular}{lllllll}
$(150,0,150)$ & 107185.2 & 99829.3 & 0.48321 & -0.16307 & -24.8739 & -0.46671 \\
$(150,50,150)$ & 107184.3 & 99830.2 & 0.473198 & -0.17136 & -24.9879 & -0.47511 \\
$(150,100,150)$ & 107185.2 & 99831.5 & 0.498778 & -0.16851 & -25.4965 & -0.48318 \\
$(150,150,150)$ & 107185.2 & 99828.7 & 0.488515 & -0.16292 & -24.8981 & -0.46447 \\
$(-50,0,-150)$ & 107188.7 & 99833.9 & 0.470915 & -0.15847 & -24.9918 & -0.48747 \\
$(-50,-50,-150)$ & 107185.2 & 99831.6 & 0.489889 & -0.16598 & -25.2295 & -0.4793 \\
$(-50,-100,-150)$ & 107184.6 & 99260.3 & 0.471789 & -0.17542 & -24.7039 & 1.4859 \\
$(-50,-150,-150)$ & 107183.4 & 99832.8 & 0.473483 & -0.16422 & -24.9814 & -0.4842 \\
$(-100,0,-150)$ & 107184.3 & 99829.1 & 0.477607 & -0.15636 & -24.9579 & -0.46521 \\
$(-100,-50,-150)$ & 107188.7 & 99826.9 & 0.505638 & -0.16943 & -25.0266 & -0.46068 \\
$(-100,-100,-150)$ & 107186.6 & 99834.3 & 0.481567 & -0.16886 & -24.9008 & -0.48352 \\
$(-100,-150,-150)$ & 107184.6 & 99829.3 & 0.479143 & -0.18187 & -25.1191 & -0.46959 \\
$(-150,0,-150)$ & 107184.6 & 99826.9 & 0.475658 & -0.16261 & -24.9124 & -0.45965 \\
$(-150,-50,-150)$ & 107185.2 & 99829.4 & 0.475882 & -0.18741 & -25.1242 & -0.47949 \\
$(-150,-100,-150)$ & 107183.4 & 99831.5 & 0.477811 & -0.17011 & -24.9309 & -0.47579 \\
$(-150,-150,-150)$ & 107184.3 & 99828.7 & 0.505052 & -0.16087 & -25.3797 & -0.47346 \\
\hline
\end{tabular}

\section{Table 10}

Pressure values for probe E at $34.4 \mathrm{~m} / \mathrm{s}$

\begin{tabular}{llccccr}
\hline Coordinate & \multicolumn{1}{c}{$P_{21}$} & $P_{22}$ & $P_{23}$ & $P_{24}$ & $P_{25}$ & $\bar{P}$ \\
\hline$(0,0,0)$ & 99984.7 & 99646.6 & 99597.2 & 99646.1 & 99786.5 & 99669.1 \\
$(50,0,0)$ & 99982 & 99644.7 & 99593.5 & 99647.3 & 99783.7 & 99667.3 \\
$(50,50,0)$ & 99983.7 & 99647.6 & 99596.3 & 99644.4 & 99789.2 & 99669.38 \\
$(50,100,0)$ & 99978.6 & 99642.3 & 99595.2 & 99646.6 & 99787.6 & 99667.93 \\
$(50,150,0)$ & 99985.2 & 99646.1 & 99593.7 & 99647.3 & 99781.9 & 99667.25 \\
$(100,0,0)$ & 99982.6 & 99647 & 99599.1 & 99646 & 99784.5 & 99669.25 \\
$(100,50,0)$ & 99978.1 & 99648.2 & 99593.7 & 99647.7 & 99786.5 & 99669.03 \\
$(100,100,0)$ & 99983.7 & 99646.3 & 99594.1 & 99643.6 & 99783.7 & 99666.93 \\
$(100,150,0)$ & 99981.5 & 99647.3 & 99599.4 & 99646.3 & 99789.2 & 99670.55 \\
$(150,0,0)$ & 99977.6 & 99648.2 & 99597.3 & 99645.4 & 99787.6 & 99669.63 \\
$(150,50,0)$ & 99983.6 & 99649.8 & 99596.7 & 99643 & 99781.9 & 99667.95 \\
$(150,100,0)$ & 99981.7 & 99648.5 & 99594.3 & 99647.6 & 99784.5 & 99668.73 \\
$(150,150,0)$ & 99983.9 & 99644.2 & 99597.6 & 99647.8 & 99785.7 & 99668.83 \\
$(-50,0,0)$ & 99979.3 & 99648.2 & 99593.6 & 99646.3 & 99787.2 & 99668.83 \\
$(-50,-50,0)$ & 99984.3 & 99649.8 & 99595.2 & 99647.1 & 99785.7 & 99669.45 \\
$(-50,-100,0)$ & 99980.7 & 99647.1 & 99593.7 & 99646.6 & 99782.8 & 99667.55 \\
$(-50,-150,0)$ & 99981.3 & 99646.6 & 99597.6 & 99644.7 & 99786.5 & 99668.85 \\
$(-100,0,0)$ & 99978.6 & 99644.7 & 99593.6 & 99646.1 & 99783.7 & 99667.03
\end{tabular}




\begin{tabular}{|c|c|c|c|c|c|c|}
\hline$(-100,-50,0)$ & 99985.2 & 99647.6 & 99595.2 & 99647.3 & 99789.2 & 99669.83 \\
\hline$(-100,-100,0)$ & 99982.6 & 99642.3 & 99593.7 & 99644.4 & 99787.6 & 99667 \\
\hline$(-100,-150,0)$ & 99978.1 & 99648.2 & 99599.1 & 99646.6 & 99781.9 & 99668.95 \\
\hline$(-150,0,0)$ & 99983.7 & 99646.3 & 99593.4 & 99647.3 & 99784.5 & 99667.88 \\
\hline$(-150,-50,0)$ & 99981.5 & 99648.5 & 99594.6 & 99646 & 99786.3 & 99668.88 \\
\hline$(-150,-100,0)$ & 99977.6 & 99644.2 & 99591.4 & 99647.7 & 99789.8 & 99668.28 \\
\hline$(-150,-150,0)$ & 99983.6 & 99644.4 & 99597 & 99643.6 & 99787.75 & 99668.24 \\
\hline$(50,0,50)$ & 99981.7 & 99646.6 & 99593.5 & 99646.3 & 99782.9 & 99667.33 \\
\hline$(50,50,50)$ & 99984.7 & 99647 & 99596.3 & 99645.4 & 99784.6 & 99668.4 \\
\hline$(50,100,50)$ & 99982 & 99648.2 & 99593.7 & 99643 & 99782.7 & 99667 \\
\hline$(50,150,50)$ & 99983.7 & 99646.3 & 99591.4 & 99647.6 & 99785.1 & 99667.6 \\
\hline$(100,0,50)$ & 99978.6 & 99647.3 & 99597.2 & 99647.8 & 99787.6 & 99669.98 \\
\hline$(100,50,50)$ & 99985.2 & 99648.2 & 99597.3 & 99646.3 & 99781.9 & 99668.43 \\
\hline$(100,100,50)$ & 99982.6 & 99649.8 & 99596.7 & 99647.1 & 99784.5 & 99669.53 \\
\hline$(100,150,50)$ & 99978.1 & 99648.5 & 99594.3 & 99646.6 & 99785.7 & 99668.78 \\
\hline$(150,0,50)$ & 99983.7 & 99644.2 & 99597.6 & 99644.7 & 99787.2 & 99668.43 \\
\hline$(150,50,50)$ & 99981.5 & 99648.2 & 99593.6 & 99647.6 & 99785.7 & 99668.78 \\
\hline$(150,100,50)$ & 99977.6 & 99649.8 & 99595.2 & 99642.3 & 99782.8 & 99667.53 \\
\hline$(150,150,50)$ & 99983.6 & 99647.1 & 99593.7 & 99646.1 & 99786.5 & 99668.35 \\
\hline$(-50,0,-50)$ & 99981.7 & 99646.6 & 99597.6 & 99647.3 & 99783.7 & 99668.8 \\
\hline$(-50,-50,-50)$ & 99983.9 & 99644.7 & 99593.6 & 99644.4 & 99789.2 & 99667.98 \\
\hline$(-50,-100,-50)$ & 99979.3 & 99647.6 & 99595.2 & 99646.6 & 99787.6 & 99669.25 \\
\hline$(-50,-150,-50)$ & 99984.7 & 99642.3 & 99594.6 & 99647.3 & 99781.9 & 99666.53 \\
\hline$(-100,0,-50)$ & 99982 & 99646.1 & 99591.4 & 99648.2 & 99784.5 & 99667.55 \\
\hline$(-100,-50,-50)$ & 99983.7 & 99649.8 & 99597 & 99644.2 & 99786.3 & 99669.38 \\
\hline$(-100,-100,-50)$ & 99978.6 & 99646 & 99593.5 & 99648.2 & 99789.8 & 99669.4 \\
\hline$(-100,-150,-50)$ & 99985.2 & 99649.8 & 99596.3 & 99649.8 & 99787.8 & 99670.91 \\
\hline$(-150,0,-50)$ & 99982.6 & 99647.1 & 99597.3 & 99647.1 & 99782.9 & 99668.6 \\
\hline$(-150,-50,-50)$ & 99978.1 & 99646.6 & 99596.7 & 99647 & 99781.5 & 99667.85 \\
\hline$(-150,-100,-50)$ & 99983.7 & 99644.7 & 99594.3 & 99644.7 & 99785.3 & 99667.25 \\
\hline$(-150,-150,-50)$ & 99981.5 & 99647.6 & 99597.6 & 99647.6 & 99785.7 & 99669.63 \\
\hline$(50,0,100)$ & 99977.6 & 99642.3 & 99593.6 & 99642.3 & 99787.2 & 99666.35 \\
\hline$(-50,50,100)$ & 99983.6 & 99648.2 & 99595.2 & 99646.1 & 99785.7 & 99668.8 \\
\hline$(-50,100,100)$ & 99984.7 & 99646.3 & 99593.7 & 99647.3 & 99782.8 & 99667.53 \\
\hline$(50,150,100)$ & 99982 & 99648.5 & 99597.6 & 99644.4 & 99786.5 & 99669.25 \\
\hline$(100,0,100)$ & 99984.7 & 99644.2 & 99593.6 & 99646.6 & 99783.7 & 99667.03 \\
\hline$(100,50,100)$ & 99982 & 99644.4 & 99595.2 & 99647.3 & 99789.2 & 99669.03 \\
\hline$(100,100,100)$ & 99983.7 & 99646.6 & 99592.4 & 99646 & 99787.6 & 99668.18 \\
\hline$(100,150,100)$ & 99978.6 & 99647 & 99595.5 & 99647.7 & 99781.9 & 99668.1 \\
\hline$(150,0,100)$ & 99985.2 & 99648.2 & 99597.1 & 99643.6 & 99784.5 & 99668.35 \\
\hline$(150,50,100)$ & 99982.6 & 99646.3 & 99596.6 & 99646.3 & 99786.3 & 99668.88 \\
\hline
\end{tabular}




\begin{tabular}{|c|c|c|c|c|c|c|}
\hline$(150,100,100)$ & 99978.1 & 99647.3 & 99593 & 99645.4 & 99789.8 & 99668.98 \\
\hline$(150,150,100)$ & 99983.7 & 99648.2 & 99593.7 & 99643 & 99787.8 & 99668.26 \\
\hline$(-50,0,-100)$ & 99981.5 & 99649.8 & 99597.6 & 99647.6 & 99782.9 & 99669.48 \\
\hline$(-50,-50,-100)$ & 99977.6 & 99648.5 & 99594.1 & 99647.8 & 99784.6 & 99668.75 \\
\hline$(-50,-100,-100)$ & 99983.6 & 99644.2 & 99599.4 & 99646.3 & 99782.7 & 99668.15 \\
\hline$(-50,-150,-100)$ & 99981.7 & 99648.2 & 99597.3 & 99647.1 & 99786.5 & 99669.78 \\
\hline$(-100,0,-100)$ & 99983.9 & 99649.8 & 99596.7 & 99646.6 & 99783.7 & 99669.2 \\
\hline$(-100,-50,-100)$ & 99979.3 & 99647.1 & 99594.3 & 99644.7 & 99789.2 & 99668.83 \\
\hline$(-100,-100,-100)$ & 99984.3 & 99646.6 & 99597.6 & 99647.6 & 99787.6 & 99669.85 \\
\hline$(-100,-150,-100)$ & 99980.7 & 99644.4 & 99593.6 & 99642.3 & 99781.9 & 99665.55 \\
\hline$(-150,0,-100)$ & 99981.3 & 99646.6 & 99595.2 & 99646.1 & 99784.5 & 99668.1 \\
\hline$(-150,-50,-100)$ & 99983.6 & 99647 & 99593.7 & 99647.3 & 99786.3 & 99668.65 \\
\hline$(-150,-100,-100)$ & 99982.2 & 99648.2 & 99599.1 & 99644.4 & 99789.8 & 99670.38 \\
\hline$(-150,-150,-100)$ & 99980.1 & 99646.3 & 99591 & 99646.6 & 99787.8 & 99668.01 \\
\hline$(50,0,150)$ & 99979.6 & 99647.3 & 99597.2 & 99647.3 & 99782.9 & 99668.68 \\
\hline$(-50,50,150)$ & 99979.3 & 99648.2 & 99593.5 & 99648.2 & 99781.5 & 99667.85 \\
\hline$(-50,100,150)$ & 99981.2 & 99649.8 & 99596.3 & 99646.3 & 99785.3 & 99669.43 \\
\hline$(50,150,150)$ & 99980.5 & 99648.5 & 99595 & 99648.5 & 99785.7 & 99669.48 \\
\hline$(100,0,150)$ & 99982.3 & 99644.2 & 99594.1 & 99644.2 & 99787.2 & 99667.43 \\
\hline$(100,50,150)$ & 99984.7 & 99648.2 & 99599.4 & 99648 & 99785.7 & 99670.38 \\
\hline$(100,100,150)$ & 99982.3 & 99649.8 & 99597.3 & 99649.8 & 99782.8 & 99669.93 \\
\hline$(100,150,150)$ & 99983.7 & 99647.1 & 99596.7 & 99646.1 & 99786.5 & 99669.1 \\
\hline$(150,0,150)$ & 99978.6 & 99646.6 & 99594.3 & 99647.3 & 99783.7 & 99667.98 \\
\hline$(150,50,150)$ & 99982.6 & 99644.7 & 99597.6 & 99644 & 99789.2 & 99668.98 \\
\hline$(150,100,150)$ & 99978.1 & 99647.6 & 99593.6 & 99646.6 & 99787.6 & 99668.85 \\
\hline$(150,150,150)$ & 99983.7 & 99642.3 & 99595.2 & 99647.3 & 99781.9 & 99666.68 \\
\hline$(-50,0,-150)$ & 99981.5 & 99646.1 & 99593.7 & 99648.2 & 99784.5 & 99668.13 \\
\hline$(-50,-50,-150)$ & 99980.7 & 99649.8 & 99599.1 & 99646.3 & 99786.3 & 99670.38 \\
\hline$(-50,-100,-150)$ & 99981.3 & 99646 & 99593.4 & 99646.1 & 99789.8 & 99668.85 \\
\hline$(-50,-150,-150)$ & 99982.5 & 99649.8 & 99594.6 & 99647.3 & 99787.8 & 99669.86 \\
\hline$(-100,0,-150)$ & 99980.9 & 99647.1 & 99591.4 & 99648.2 & 99782.9 & 99667.4 \\
\hline$(-100,-50,-150)$ & 99981.3 & 99644.2 & 99597 & 99646.3 & 99784.5 & 99668.05 \\
\hline$(-100,-100,-150)$ & 99980.7 & 99644.4 & 99593.5 & 99648.5 & 99786.3 & 99668.18 \\
\hline$(-100,-150,-150)$ & 99980 & 99646.6 & 99596.3 & 99644.2 & 99789.8 & 99669.23 \\
\hline$(-150,0,-150)$ & 99982.7 & 99647 & 99599.1 & 99648.2 & 99787.8 & 99670.59 \\
\hline$(-150,-50,-150)$ & 99983.7 & 99648.2 & 99593 & 99649.8 & 99782.9 & 99668.58 \\
\hline$(-150,-100,-150)$ & 99980.3 & 99646.3 & 99594.6 & 99648.2 & 99781.5 & 99667.65 \\
\hline$(-150,-150,-150)$ & 99977.4 & 99647.3 & 99591.4 & 99646.3 & 99785.3 & 99667.58 \\
\hline
\end{tabular}

\section{Table 11}


Pressure Coefficient values for probe E at $34.4 \mathrm{~m} / \mathrm{s}$

\begin{tabular}{|c|c|c|c|c|c|c|}
\hline Coordinate & $P_{\text {total (pitot) }}$ & $P_{\text {static (pitot) }}$ & $C_{P_{\text {yaw }}}$ & $C_{P_{\text {pitch }}}$ & $C_{P_{\text {total }}}$ & $C_{P_{\text {static }}}$ \\
\hline$(0,0,0)$ & 107185.2 & 99829.3 & 0.156527 & 0.444867 & -22.8153 & -0.5076 \\
\hline$(50,0,0)$ & 107183.4 & 99825.7 & 0.16254 & 0.433016 & -22.8606 & -0.50286 \\
\hline$(50,50,0)$ & 107187.6 & 99827.3 & 0.163207 & 0.46067 & -22.9186 & -0.50243 \\
\hline$(50,100,0)$ & 107184.6 & 99822.8 & 0.151605 & 0.45385 & -23.1947 & -0.49851 \\
\hline$(50,150,0)$ & 107183.6 & 99823.7 & 0.164806 & 0.423337 & -22.64 & -0.49206 \\
\hline$(100,0,0)$ & 107184.7 & 99829.3 & 0.153822 & 0.441679 & -22.9842 & -0.51077 \\
\hline$(100,50,0)$ & 107184.6 & 99830.2 & 0.176333 & 0.449082 & -23.3163 & -0.52148 \\
\hline$(100,100,0)$ & 107185.0 & 99831.5 & 0.164786 & 0.44227 & -22.7332 & -0.51953 \\
\hline$(100,150,0)$ & 107183.4 & 99828.7 & 0.154044 & 0.459559 & -23.1608 & -0.5086 \\
\hline$(150,0,0)$ & 107184.3 & 99833.9 & 0.165273 & 0.461726 & -23.4003 & -0.5334 \\
\hline$(150,50,0)$ & 107188.7 & 99831.6 & 0.168224 & 0.438777 & -22.8262 & -0.51845 \\
\hline$(150,100,0)$ & 107186.6 & 99260.3 & 0.173177 & 0.437415 & -23.0207 & 1.304976 \\
\hline$(150,150,0)$ & 107184.6 & 99832.8 & 0.147901 & 0.437674 & -22.8539 & -0.52043 \\
\hline$(-50,0,0)$ & 107182.0 & 99829.1 & 0.17586 & 0.453821 & -23.199 & -0.51623 \\
\hline$(-50,-50,0)$ & 107187.1 & 99826.9 & 0.173416 & 0.44021 & -22.8769 & -0.50008 \\
\hline$(-50,-100,0)$ & 107184.6 & 99834.3 & 0.170525 & 0.434935 & -23.0046 & -0.53249 \\
\hline$(-50,-150,0)$ & 107183.6 & 99829.3 & 0.156825 & 0.453833 & -23.051 & -0.51352 \\
\hline$(-100,0,0)$ & 107184.7 & 99826.9 & 0.164005 & 0.441627 & -23.128 & -0.51312 \\
\hline$(-100,-50,0)$ & 107184.6 & 99829.4 & 0.166151 & 0.449941 & -22.8281 & -0.50598 \\
\hline$(-100,-100,0)$ & 107183.4 & 99831.5 & 0.153992 & 0.453739 & -22.8161 & -0.52123 \\
\hline$(-100,-150,0)$ & 107184.3 & 99828.7 & 0.158823 & 0.437652 & -23.3097 & -0.51674 \\
\hline$(-150,0,0)$ & 107188.7 & 99833.9 & 0.167498 & 0.434418 & -22.8133 & -0.52569 \\
\hline$(-150,-50,0)$ & 107186.6 & 99831.6 & 0.172411 & 0.448461 & -23.0471 & -0.52051 \\
\hline$(-150,-100,0)$ & 107184.6 & 99260.3 & 0.170694 & 0.459387 & -23.2991 & 1.31892 \\
\hline$(-150,-150,0)$ & 107184.6 & 99832.8 & 0.149669 & 0.457093 & -22.834 & -0.52182 \\
\hline$(50,0,50)$ & 107185.2 & 99834.3 & 0.168907 & 0.434513 & -22.9138 & -0.53113 \\
\hline$(50,50,50)$ & 107183.4 & 99833.9 & 0.161239 & 0.440089 & -22.759 & -0.52324 \\
\hline$(50,100,50)$ & 107184.3 & 99832.8 & 0.172851 & 0.441801 & -22.8417 & -0.52585 \\
\hline$(50,150,50)$ & 107188.7 & 99832.8 & 0.173679 & 0.434989 & -22.7934 & -0.52262 \\
\hline$(100,0,50)$ & 107186.6 & 99831.6 & 0.162333 & 0.452977 & -23.3552 & -0.52369 \\
\hline$(100,50,50)$ & 107184.6 & 99831.5 & 0.160682 & 0.428064 & -22.7272 & -0.5148 \\
\hline$(100,100,50)$ & 107185.2 & 99829.4 & 0.169608 & 0.438872 & -23.0061 & -0.51066 \\
\hline$(100,150,50)$ & 107185.2 & 99829.3 & 0.17522 & 0.449689 & -23.2995 & -0.51895 \\
\hline$(150,0,50)$ & 107185.2 & 99829.1 & 0.147807 & 0.451986 & -22.842 & -0.50963 \\
\hline$(150,50,50)$ & 107185.2 & 99828.7 & 0.174594 & 0.441602 & -23.0353 & -0.51139 \\
\hline$(150,100,50)$ & 107184.6 & 99826.9 & 0.176086 & 0.453116 & -23.2428 & -0.51399 \\
\hline$(150,150,50)$ & 107183.4 & 99826.9 & 0.169389 & 0.445361 & -22.8383 & -0.50293 \\
\hline$(-50,0,-50)$ & 107184.3 & 99260.3 & 0.1566 & 0.435922 & -23.0189 & 1.305529 \\
\hline
\end{tabular}




\begin{tabular}{|c|c|c|c|c|c|c|}
\hline$(-50,-50,-50)$ & 107188.7 & 99834.3 & 0.161747 & 0.458337 & -22.8054 & -0.52647 \\
\hline$(-50,-100,-50)$ & 107186.6 & 99834.3 & 0.169005 & 0.454765 & -23.2456 & -0.53233 \\
\hline$(-50,-150,-50)$ & 107184.6 & 99833.9 & 0.149917 & 0.423038 & -22.6287 & -0.52605 \\
\hline$(-100,0,-50)$ & 107184.6 & 99833.9 & 0.173789 & 0.433042 & -22.8826 & -0.52851 \\
\hline$(-100,-50,-50)$ & 107185.2 & 99832.8 & 0.167343 & 0.45208 & -22.9111 & -0.51992 \\
\hline$(-100,-100,-50)$ & 107183.4 & 99832.8 & 0.170116 & 0.457956 & -23.3013 & -0.52846 \\
\hline$(-100,-150,-50)$ & 107184.3 & 99832.8 & 0.170226 & 0.438929 & -22.9061 & -0.51509 \\
\hline$(-150,0,-50)$ & 107188.7 & 99831.6 & 0.158599 & 0.432484 & -22.9494 & -0.51911 \\
\hline$(-150,-50,-50)$ & 107185.2 & 99831.6 & 0.160838 & 0.434811 & -23.2301 & -0.5278 \\
\hline$(-150,-100,-50)$ & 107184.6 & 99831.5 & 0.159267 & 0.444304 & -22.7553 & -0.51904 \\
\hline$(-150,-150,-50)$ & 107183.4 & 99825.7 & 0.160321 & 0.442806 & -23.0921 & -0.50044 \\
\hline$(50,0,100)$ & 107184.3 & 99827.3 & 0.156466 & 0.465542 & -23.1541 & -0.51711 \\
\hline$(-50,50,100)$ & 107188.7 & 99822.8 & 0.168361 & 0.443456 & -22.8879 & -0.4892 \\
\hline$(-50,100,100)$ & 107186.6 & 99823.7 & 0.165839 & 0.427209 & -22.7064 & -0.49239 \\
\hline$(50,150,100)$ & 107184.6 & 99829.3 & 0.162594 & 0.453921 & -23.0069 & -0.51126 \\
\hline$(100,0,100)$ & 107184.6 & 99830.2 & 0.159282 & 0.431573 & -22.6644 & -0.51365 \\
\hline$(100,50,100)$ & 107185.2 & 99831.5 & 0.157051 & 0.452957 & -22.9923 & -0.51863 \\
\hline$(100,100,100)$ & 107183.4 & 99828.7 & 0.171777 & 0.448459 & -22.818 & -0.50876 \\
\hline$(100,150,100)$ & 107184.3 & 99833.9 & 0.166828 & 0.432206 & -23.2068 & -0.53398 \\
\hline$(150,0,100)$ & 107188.7 & 99831.6 & 0.161275 & 0.44469 & -22.7347 & -0.51523 \\
\hline$(150,50,100)$ & 107184.3 & 99260.3 & 0.158419 & 0.446251 & -22.9555 & 1.302335 \\
\hline$(150,100,100)$ & 107188.7 & 99832.8 & 0.174363 & 0.467125 & -23.3258 & -0.52996 \\
\hline$(150,150,100)$ & 107186.6 & 99829.1 & 0.172776 & 0.457618 & -22.8346 & -0.50989 \\
\hline$(-50,0,-100)$ & 107184.6 & 99826.9 & 0.167294 & 0.433619 & -23.085 & -0.50453 \\
\hline$(-50,-50,-100)$ & 107184.6 & 99834.3 & 0.176137 & 0.442933 & -23.335 & -0.53602 \\
\hline$(-50,-100,-100)$ & 107185.2 & 99829.3 & 0.142019 & 0.432398 & -22.8297 & -0.51086 \\
\hline$(-50,-150,-100)$ & 107183.4 & 99826.9 & 0.16318 & 0.446902 & -23.0878 & -0.50373 \\
\hline$(-100,0,-100)$ & 107184.3 & 99829.4 & 0.168732 & 0.435653 & -22.8802 & -0.50906 \\
\hline$(-100,-50,-100)$ & 107188.7 & 99831.5 & 0.170062 & 0.465416 & -23.2205 & -0.52396 \\
\hline$(-100,-100,-100)$ & 107185.2 & 99828.7 & 0.155828 & 0.445222 & -22.9001 & -0.50517 \\
\hline$(-100,-150,-100)$ & 107188.7 & 99833.9 & 0.161193 & 0.442964 & -22.8716 & -0.53419 \\
\hline$(-150,0,-100)$ & 107185.2 & 99826.9 & 0.164112 & 0.44189 & -23.001 & -0.50702 \\
\hline$(-150,-50,-100)$ & 107184.6 & 99832.8 & 0.170186 & 0.44134 & -22.8639 & -0.52119 \\
\hline$(-150,-100,-100)$ & 107183.4 & 99832.8 & 0.15746 & 0.466287 & -23.0936 & -0.52089 \\
\hline$(-150,-150,-100)$ & 107184.3 & 99831.6 & 0.175912 & 0.452277 & -23.0839 & -0.52417 \\
\hline$(50,0,150)$ & 107188.7 & 99831.5 & 0.161132 & 0.436118 & -23.186 & -0.52368 \\
\hline$(-50,50,150)$ & 107186.6 & 99829.4 & 0.17563 & 0.427998 & -23.1411 & -0.5187 \\
\hline$(-50,100,150)$ & 107184.6 & 99829.4 & 0.171598 & 0.445834 & -23.1045 & -0.51311 \\
\hline$(50,150,150)$ & 107184.6 & 99829.3 & 0.171369 & 0.441122 & -23.1624 & -0.51387 \\
\hline$(100,0,150)$ & 107185.2 & 99825.7 & 0.159111 & 0.454148 & -22.8755 & -0.50266 \\
\hline$(100,50,150)$ & 107183.4 & 99827.3 & 0.155253 & 0.437445 & -22.902 & -0.49924 \\
\hline
\end{tabular}




\begin{tabular}{lllllll}
$(100,100,150)$ & 107184.3 & 99822.8 & 0.168067 & 0.42577 & -23.0556 & -0.4894 \\
$(100,150,150)$ & 107188.7 & 99823.7 & 0.160203 & 0.446281 & -22.9021 & -0.49142 \\
$(150,0,150)$ & 107185.2 & 99829.3 & 0.16837 & 0.439115 & -23.2004 & -0.51936 \\
$(150,50,150)$ & 107184.3 & 99830.2 & 0.150179 & 0.461698 & -22.9628 & -0.51407 \\
$(150,100,150)$ & 107185.2 & 99831.5 & 0.174616 & 0.455942 & -23.3052 & -0.52595 \\
$(150,150,150)$ & 107185.2 & 99828.7 & 0.148569 & 0.424572 & -22.716 & -0.51108 \\
$(-50,0,-150)$ & 107188.7 & 99833.9 & 0.167212 & 0.434942 & -22.9986 & -0.529 \\
$(-50,-50,-150)$ & 107185.2 & 99831.6 & 0.163377 & 0.45114 & -23.2161 & -0.51954 \\
$(-50,-100,-150)$ & 107184.6 & 99260.3 & 0.168667 & 0.459914 & -23.0542 & 1.307569 \\
$(-50,-150,-150)$ & 107183.4 & 99832.8 & 0.176562 & 0.449242 & -23.0326 & -0.52117 \\
$(-100,0,-150)$ & 107184.3 & 99829.1 & 0.177671 & 0.429665 & -22.9774 & -0.51579 \\
$(-100,-50,-150)$ & 107188.7 & 99826.9 & 0.15004 & 0.441181 & -23.0085 & -0.5071 \\
$(-100,-100,-150)$ & 107186.6 & 99834.3 & 0.162867 & 0.440925 & -23.057 & -0.53156 \\
$(-100,-150,-150)$ & 107184.6 & 99829.3 & 0.162062 & 0.46911 & -23.2139 & -0.51575 \\
$(-150,0,-150)$ & 107184.6 & 99826.9 & 0.154431 & 0.447114 & -23.0747 & -0.50082 \\
$(-150,-50,-150)$ & 107185.2 & 99829.4 & 0.173899 & 0.422372 & -22.8529 & -0.51035 \\
$(-150,-100,-150)$ & 107183.4 & 99831.5 & 0.165361 & 0.426355 & -23.0387 & -0.52407 \\
$(-150,-150,-150)$ & 107184.3 & 99828.7 & 0.180424 & 0.44864 & -23.2612 & -0.52005 \\
\hline
\end{tabular}

